\title{
QUANTITATIVE LANGUAGES DEFINED BY FUNCTIONAL AUTOMATA*
}

\author{
EMMANUEL FILIOT $^{a}$, RAFFAELLA GENTILINI $^{b}$, AND JEAN-FRANÇOIS RASKIN $^{c}$ \\ ${ }^{a, c}$ Université Libre de Bruxelles, Computer Science Department \\ e-mail address: \{efilio, jraskin\}t@ulb.ac.be \\ ${ }^{b}$ University of Perugia, Department of Mathematics and Computer Science \\ e-mail address: raffaella.gentilini@dmi.unipg.it
}

\begin{abstract}
A weighted automaton is functional if any two accepting runs on the same finite word have the same value. In this paper, we investigate functional weighted automata for four different measures: the sum, the mean, the discounted sum of weights along edges and the ratio between rewards and costs. On the positive side, we show that functionality is decidable for the four measures. Furthermore, the existential and universal threshold problems, the language inclusion problem and the equivalence problem are all decidable when the weighted automata are functional. On the negative side, we also study the quantitative extension of the realizability problem and show that it is undecidable for sum, mean and ratio. We finally show how to decide whether the language associated with a given functional automaton can be defined with a deterministic one, for sum, mean and discounted sum. The results on functionality and determinizability are expressed for the more general class of functional group automata. This allows one to formulate within the same framework new results related to discounted sum automata and known results on sum and mean automata. Ratio automata do not fit within this general scheme and different techniques are required to decide functionality.
\end{abstract}

\section{INTRODUCTION}

Recently, there have been several efforts made to lift the foundations of computer aided verification and synthesis from the basic Boolean case to the richer quantitative case, e.g. [11, 8, 2]. This paper belongs to this line of research and contributes to the study of quantitative languages over finite words.

2012 ACM CCS: [Theory of computation]: Formal languages and automata theory.

Key words and phrases: quantitative languages, weighted automata, functionality, realizability, determinization.

* This work is a revised and extended version of [19].

${ }^{a}$ Emmanuel Filiot is supported by F.R.S.-FNRS (as a research associate, "Chercheur Qualifié").

${ }^{b}$ Raffaella Gentilini acknowledges support from the Italian National Group for Scientific Computing (GNCS-INdAM).

${ }^{c}$ Jean-François Raskin is supported by ERC Starting Grant (279499: inVEST). 
Our paper proposes a systematic study of the algorithmic properties of several classes of functional weighted automata (defining quantitative languages). A functional weighted automaton is a nondeterministic weighted automaton such that any two accepting runs $\rho_{1}$, $\rho_{2}$ on a word $w$ associate with this word a unique value $\mathrm{V}\left(\rho_{1}\right)=\mathrm{V}\left(\rho_{2}\right)$. As we show in this paper, several important verification problems are decidable for nondeterministic functional weighted automata while they are undecidable (or not known to be decidable) for the full class of nondeterministic weighted automata.

In this paper, we study automata in which an integer weight, or a pair of integer weights, is associated with each of their transitions. From those weights, an (accepting) run $\rho$ on a word $w$ associates a sequence of weights with the word, and this sequence is mapped to a rational value by a measure function. We consider four different measure functions: (i) Sum computes the sum of the weights along the sequence, (ii) Avg returns the mean value of the weights, (iii) Dsum computes the discounted sum of the weights for a given discount factor $\lambda \in \mathbb{Q} \cap] 0,1[$, and (iv) Ratio is applied to a sequence of pairs of weights, and it returns the ratio between the sum of weights appearing as the first component (rewards) and the sum of the weights appearing as the second component (costs). All those measures are motivated by applications in computer aided verification and synthesis, see for example [14, 7]. The value associated with a word $w$ is obtained by combining all the values of the accepting runs on $w$ with a particular operation (usually max or min). The value of $w$ is denoted by $L_{A}(w)$.

Contributions Classical results on weighted automata consider operations over semirings: the value of a run is obtained as the multiplication of the values along its transitions, and the values of all runs on the same input word are combined with addition [16]. Since we focus on functional automata, all the accepting runs have the same value, and so we do not need addition. Whenever it is possible, we phrase our results in the general framework of functional group automata, i.e. automata whose transitions are weighted by elements of a group. In particular, Sum, Avg, and Dsum can be seen as operations over a group. For Ratio however, we always need different techniques, and leave its encoding in terms of a group operation as open.

We first show that functionality is decidable in PTime for group automata (operations on group elements are assumed to be computable in polynomial time). This implies that functionality is PTime for Dsum automata and generalizes known results for Sum and Avg automata. By using a pumping argument, we show that functionality is in CoNP for Ratio-automata.

Then we solve the following decision problems, along the line of [11]. First, we consider threshold problems. The existential (universal, respectively) threshold problem asks, given a weighted automaton $A$ and a threshold $\nu \in \mathbb{Q}$, if there exists a word (if for all words, respectively) $w$ accepted by $A: L_{A}(w) \geq \nu$. Those problems can be seen as generalizations of the emptiness and universality problems for finite-state automata. Second, we consider the quantitative language inclusion problem that asks, given two weighted automata $A$ and $B$, if all words accepted by $A$ are also accepted by $B$, and for all accepted words $w$ of $A$, we have $L_{A}(w) \leq L_{B}(w)$. We show that all those problems are decidable for the four classes of measure functions that we consider in this paper when the automata are functional. In

\footnotetext{
${ }^{1}$ We do not consider the measure functions Min and Max that map a sequence to the minimal and the maximal value that appear in the sequence as the nondeterministic automata that use those measure functions can be made deterministic and all the decision problems for them have known and simple solutions [1].
} 
particular, for Ratio, we show decidability of the inclusion problem using a recent algorithm to solve quadratic diophantine equations [20], this is a new deep result in mathematics and the complexity of the algorithm is not yet known. We also show that the equivalence problem can be decided in polynomial space for Ratio via an easy reduction to functionality. Note that those decidability results are in sharp contrast with the corresponding results for the full class of nondeterministic weighted automata: for that class, only the existential threshold problem is known to be decidable, the language inclusion problem is undecidable for Sum [25, 1], and therefore for Avg and Ratio, while the problem is open for Dsum.

Finally, we consider a (finite word) quantitative variant of the realizability problem introduced by Church, which is related to the synthesis of reactive systems [32, 35] and can be formalized as a game in which two players (the system and the environment) alternate in choosing letters in their respective alphabet of signals. The system can decide to stop the game. By doing so, they form a word which is obtained by concatenating the successive choices of the players. The realizability problem asks, given a weighted automaton $A$, a threshold $\nu \geq 0$, and an alphabet $\Sigma=\Sigma_{1} \times \Sigma_{2}$, if there exists a strategy for choosing the letters in $\Sigma_{1}$ in the word forming game such that no matter how the adversary chooses his letters in $\Sigma_{2}$, the word $w$ that is obtained belongs to the language of $A$ and $A(w)>\nu$. We show that this problem is undecidable for Sum, Avg, and Ratio functional automata (the case Dsum is left open). However, we show that the realizability problem is decidable for the deterministic versions of the automata studied in this paper. This motivates the determinizability problem.

The determinizability problem asks, given a functional weighted automaton $A$, if the quantitative language defined by $A$ is also definable by a deterministic automaton. It is known that Sum, Avg and Dsum-automata are not determinizable in general [11]. We give here a decidable necessary and sufficient condition for the determinizability of functional group automata, and we show how to construct a deterministic automaton from the functional one when this is possible. As a corollary, we obtain a decidable characterization of determinizable functional Sum-, Avg- and Dsum-automata. While it was known for Sum (and as a consequence for Avg, by seing the Avg-automaton as a Sum-automaton) [23], it is new for Dsum.

Functionality versus Unambiguity Functional weighted automata are a natural generalization of unambiguous weighted automata, i.e. weighted automata such that there is at most one accepting run for each input word. Since unambiguity captures most of the nondeterminism that is useful in practice, our results are both theoretically and practically important. Functional weighted automata are equivalent (modulo an exponential blow-up) to unambiguous weighted automata. This exponential blow-up is worst-case unavoidable. It is already unavoidable when going from a non-deterministic to an unambiguous finite automaton [33]. Therefore, we inherit this succinctness result.

Having algorithms to test for functionality has the nice consequence of providing algorithms to test for equivalence of functional (and hence unambiguous) weighted automata: given two functional weighted automata $A_{1}$ and $A_{2}$, they are equivalent iff they have the same domain, and their union is functional. For Ratio-automata for instance, this gives better complexity than applying our result on inclusion (which is, in some sense, a harder problem).

In all the decision problems we consider but functionality, considering unambiguous weighted automata instead of functional ones would not have simplified the algorithms nor 
the proofs, because in a way, unambiguous automata capture already all the difficulty of non-deterministic behaviours, and are, therefore, as difficult as functional automata to deal with. Therefore, considering functional automata with respect to decision problems comes, in some sense, for free.

Related Works Motivated by open problems in computer-aided verification, our work follows the same line as [11]. However [11] is concerned with weighted automata on infinite words, either non-deterministic, for which some important problems are undecidable (e.g. inclusion of Avg-automata), or deterministic ones, which are strictly less expressive than functional automata. The Ratio measure is not considered either. Their domains of quantitative languages are assumed to be total (as all states are accepting and their transition relation is total) while we can define partial quantitative languages thanks to an acceptance condition. Functional weighted automata where all states are accepting correspond to deterministic weighted automata: all the transitions that leave the same state on the same input symbol must carry the same weight, and therefore, a simple subset construction allows one to transform any such functional automaton into a deterministic one.

Except for realizability, our results for Sum-automata (and to some extent Avg-automata) are not new [30. Functionality is known to be in PTime [23, and emptiness, inclusion, equivalence (for functional Sum-automata) are already known to be decidable [26, 27]. Moreover, it is known that determinizability of functional Sum-automata is decidable in PTime [23], as well as for the strictly more expressive class of polynomially ambiguous Sum-automata [22, for which the termination of Mohri's determinization algorithm [16] is decidable. Weighted automata over semirings have been extensively studied [16], and more generally rational series [5]. Mohri's determinization algorithm has been generalized in [23] to arbitrary semirings, in which a general condition for its termination, generalizing the notion of twins property in [13, 29], is given. However, this sufficient condition only applies to commutative semirings, and therefore cannot directly be used for Dsum-automata. We rephrase the twinning property on groups that are not necessarily commutative and prove that it is a sufficient condition for a functional group automata to be determinizable. Our determinization algorithm for functional group automata is similar to Mohri's algorithm and is, in that sense, not new. However, since we cannot rely on the additive operation, we need some different computation of the output values of the constructed deterministic automaton. Further, we extend [23] from functional Sum-automata to functional Avg and Dsum-automata, proving that the twinning property is also a necessary condition toward determinization for these measures.

The techniques we use for deciding functionality and determinization are also inspired by techniques from word transducers [34, 6, 4, 13, 37]. In particular, our procedure to decide functionality of weighted automata also allows us to decide functionality of a word transducer, seen as a weighted automaton over the free group. It generalizes to arbitrary groups the procedure of [4] which was used to show that functionality of word transducers is in PTime. As in [4], it relies on a notion of delay between two runs. This notion of delay is also used for the determinization of group automata.

In [10], Boker et. al. show that Dsum-automata on infinite words with a trivial accepting condition (all states are accepting), but not necessarily functional, are determinizable for any discount factor of the form $1 / n$ for some $n \in \mathbb{N}_{\geq 2}$, while we consider arbitrary discounted factors. Their proof is based on a notion of recoverable gap, similar to that of delays. Finally in [17], the relation between discounted weighted automata over a semiring and weighted logics is studied. 
To the best of our knowledge, our results on Dsum and Ratio-automata, as well as on the realizability problem, are new. Our main and most technical results are functionality and inclusion of Ratio-automata, undecidability of the realizability of functional Sumautomata, and solvability of the deterministic versions of the realizability problem. The latter reduce to games on graphs that are to the best of our knowledge new, namely finite Sum, Avg, Dsum, Ratio-games on weighted graphs with a combination of a reachability objective and a quantitative objective.

\section{Quantitative Languages and Functionality}

Let $\Sigma$ be a finite alphabet. We denote by $\Sigma^{*}$ the set of finite words over $\Sigma$, and $\Sigma^{+}$the set of non-empty words over $\Sigma$. The empty word is denoted by $\epsilon$. In this paper, we assume that weighted automata process input words that end with a terminal symbol $\dashv \in \Sigma$. We denote by $\Sigma_{\dashv}^{+}$the set of words of the form $u \dashv$, where $u \in(\Sigma \backslash\{\dashv\})^{*}$.

A quantitative language $L$ over $\Sigma$ is a mapping $L: \Sigma_{\dashv}^{+} \rightarrow \mathbb{Q} \cup\{\perp\}$. For all $w \in \Sigma_{\dashv}^{+}$, $L(w)$ is called the value of $w . L(w)=\perp$ means that the value of $w$ is undefined. We set $\perp<v$ for all $v \in \mathbb{Q}$.

Let $n \geq 0$. Given a finite sequence $v=v_{0} \ldots v_{n}$ of integers (resp. a finite sequence $v^{\prime}=\left(r_{0}, c_{0}\right) \ldots\left(r_{n}, c_{n}\right)$ of pairs of natural numbers, $c_{i}>0$ for all $\left.i\right)$ and $\lambda \in \mathbb{Q}$ such that $0<\lambda<1$, we define the following functions:

$$
\operatorname{Sum}(v)=\sum_{i=0}^{n} v_{i} \quad \operatorname{Avg}(v)=\frac{\operatorname{Sum}(v)}{n+1} \quad \operatorname{Dsum}(v)=\sum_{i=0}^{n} \lambda^{i} v_{i} \quad \operatorname{Ratio}\left(v^{\prime}\right)=\frac{\sum_{i=0}^{n} r_{i}}{\sum_{i=0}^{n} c_{i}}
$$

Weighted Automata Let $V \in\{$ Sum, Avg, Dsum, Ratio $\}$. A weighted $V$-automaton over $\Sigma$ is a tuple $A=\left(Q, q_{I}, F, \delta, \gamma\right)$ where $Q$ is a finite set of states, $q_{I}$ is an initial state, $F$ is a set of final states, $\delta=\delta^{\prime} \cup \delta_{\dashv}$ is a transition relation, where $\delta^{\prime} \subseteq(Q \backslash F) \times(\Sigma \backslash\{\dashv\}) \times(Q \backslash F)$ and $\delta_{\dashv} \subseteq$ $(Q \backslash F) \times\{\dashv\} \times F$ is the terminal set of transitions, and $\gamma: \delta \rightarrow \mathbb{Z}$ (resp. $\gamma: \delta \rightarrow \mathbb{N} \times(\mathbb{N}-0)$ if $V=$ Ratio) is a weight function. The size of $A$ is defined by $|A|=|Q|+|\delta|+\sum_{t \in \delta} \log _{2}(\gamma(t))$. Note that $\left(Q, q_{I}, F, \delta\right)$ is a classical finite-state automaton, called the input-automaton. We say that $A$ is deterministic if its input-automaton $\left(Q, q_{I}, F, \delta\right)$ is deterministic. In the sequel, we use the term $V$-automata to denote either Sum, Dsum, Avg or Ratio-automata.

A run $\rho$ of $A$ over $w=\sigma_{0} \ldots \sigma_{n} \in \Sigma^{+}$is a sequence $\rho=q_{0} \sigma_{0} q_{1} \ldots \sigma_{n} q_{n+1}$ such that $q_{0}=q_{I}$ and for all $i \in\{0, \ldots, n\},\left(q_{I}, \sigma_{i}, q_{i+1}\right) \in \delta$. It is accepting if $q_{n+1} \in F$. We write $\rho: q_{0} \stackrel{w}{\rightarrow} q_{n+1}$ to denote that $\rho$ is a run on $w$ starting at $q_{0}$ and ending in $q_{n+1}$. Given $i \in\{0, \ldots, n\}$, we write $\rho_{i}$ to denote the prefix of the run $\rho$ until position $i$. The domain of $A$, denoted by $\operatorname{dom}(A)$, is defined as the set of words $w \in \Sigma^{*}$ on which there exists some accepting run of $A$. Note that by definition of $\delta$, we have $\operatorname{dom}(A) \subseteq \Sigma_{\dashv}^{+}=(\Sigma \backslash\{\dashv\})^{*} \dashv$. We say that $A$ is unambiguous if it admits at most one accepting run for each word.

The function $V$ is naturally extended to runs as follows:

$$
V(\rho)= \begin{cases}V\left(\gamma\left(q_{0}, \sigma_{0}, q_{1}\right) \ldots \gamma\left(q_{n}, \sigma_{n}, q_{n+1}\right)\right) & \text { if } \rho \text { is accepting } \\ \perp & \text { otherwise }\end{cases}
$$

The relation $R_{A}^{V}=\left\{(w, V(\rho)) \mid w \in \Sigma^{+}, \rho\right.$ is an accepting run of $A$ on $\left.w\right\}$ is called the relation induced by $A$. It is functional if for all words $w \in \Sigma_{-}^{+}$, we have $\left|\left\{v \mid(w, v) \in R_{A}^{V}\right\}\right| \leq$

\footnotetext{
${ }^{2}$ As in 11, we do not consider the empty word as our weighted automata do not have initial and final weight functions. This eases our presentation but all our results carry over to the more general setting with initial and final weight function [16].
} 
1. In that case we say that $A$ is functional. The quantitative language $L_{A}: \Sigma_{\dashv}^{+} \rightarrow \mathbb{Q} \cup\{\perp\}$ defined by $A$ is defined by $L_{A}: w \mapsto \max \left\{v \mid(w, v) \in R_{A}^{V}\right\}$ where $\max \emptyset=\perp$.

Example 2.1. Fig. 1 illustrates two Sum-automata over the alphabet $\{a, b, \dashv\}$. The first automaton (on the left) defines the quantitative language $w \in \Sigma_{\dashv}^{+} \mapsto \max \left(\#_{a}(w), \#_{b}(w)\right)$, where $\#_{\alpha}(w)$ denotes the number of occurrences of the letter $\alpha$ in $w$. Its induced relation is $\left\{\left(w, \#_{a}(w)\right) \mid w \in \Sigma_{\dashv}^{+}\right\} \cup\left\{\left(w, \#_{b}(w)\right) \mid w \in \Sigma_{\dashv}^{+}\right\}$. The second automaton (on the right) defines the quantitative language that maps any word of length at least 2 to the number of occurrences of its last letter but one.

Remark 2.2. In the literature, weighted automata are sometimes equipped with a terminal function that associates with accepting states a value. Instead, we assume that weighted automata accepts only words that end with the terminal symbol $\dashv$. The two models are equivalent in the following sense: we can always transform a weighted automaton $A$ over an alphabet $\Gamma$ with a terminal function, into a weighted automaton $A_{\dashv}$ over the alphabet $\Gamma \cup\{\dashv\}$ without terminal function, such that $\operatorname{dom}\left(A_{\dashv}\right)=\operatorname{dom}(A) \dashv$ and such that all the decision problems we consider are preserved, such as functionality, emptiness, determinizability, etc. In other words, $A$ is functional iff $A_{\dashv}$ is, etc. This is done by adding transitions from accepting states $q_{f}$ of $A$ to a fresh accepting state of $A_{\dashv}$ on reading $\dashv$, with values $t\left(q_{f}\right)$ if $t$ is the terminal function. We rather use terminal symbols instead of terminal functions since it lightens the notations in our proofs.

All our results but determinizability hold true for weighted automata without terminal function nor terminal symbol, because the latter can easily be encoded as weighted automata with a terminal symbol, while preserving the properties we are interested in. For determinization however, our determinization procedure, if it terminates, does not necessarily produce a deterministic weighted automaton without terminal function/symbol, even if we apply it to a weighted automaton without terminal function/symbol. The deterministic weighted automata that are constructed by our procedure coincide with the classical notion of subsequential weighted automata with terminal function that is sometimes used in the literature [16. Without terminal function/symbol, deterministic weighted automata coincide with sequential weighted automata. Since the notion of subsequentiality is more general than sequentiality, and since it already guarantees decidability of the realizability problem, we rather consider this more general class.

A state $q$ is accessible (by some word $w \in \Sigma^{*}$ ) if $A$ admits a run $\rho: q_{I} \stackrel{w}{\rightarrow} q$. A state $q$ is co-accessible (by some word $w \in \Sigma^{*}$ ) if $A$ admits a run $\rho: q \stackrel{w}{\rightarrow} q_{f}$ for some $q_{f} \in F$. We say that a state $q$ is useful if it is both accessible and co-accessible (and useless, otherwise). Useless states can be removed from the given weighted automaton in linear time, without changing the recognized language [30]. A weighted automaton with no useless state is said to be trim.

A pair of states $\left(q, q^{\prime}\right)$ is co-accessible if there exists a word $w$ such that $q$ and $q^{\prime}$ are co-accessible by $w$.

Functional Weighted Automata The Sum-automaton on the left of Fig. 1 is not functional (e.g. the word $a b b \dashv$ maps to the values 1 and 2), while the one on the right is functional (and even unambiguous). Concerning the expressiveness of functional automata, we can show that deterministic automata are strictly less expressive than functional automata which are again strictly less expressive than the full class of weighted automata. Let $V \in\{$ Sum, Avg, Ratio\}. The automata of Fig. 1 can be seen as $V$-automata (with a 

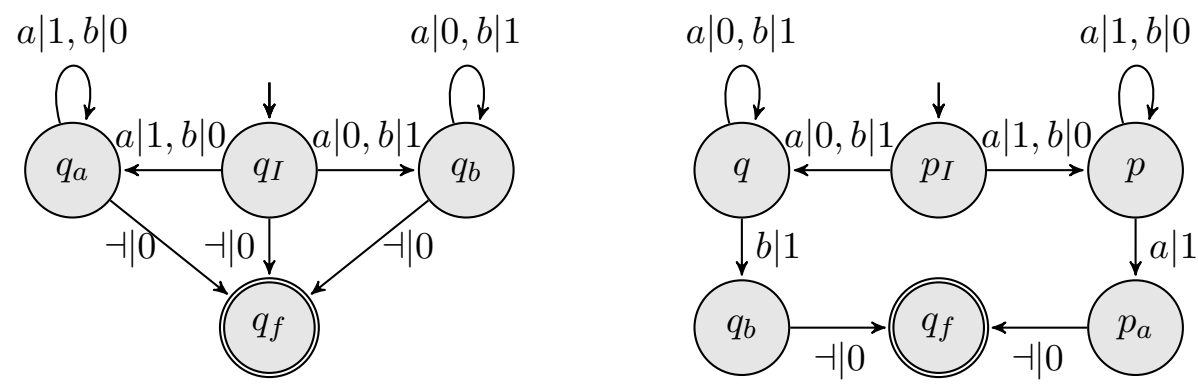

Figure 1: Examples of Sum-automata

constant cost 1 if $V=$ Ratio). The right $V$-automaton cannot be expressed by any deterministic $V$-automaton because the value of a word depends on its last letter. The left $V$-automaton cannot be expressed by any functional $V$-automaton. It is easy to verify that the above results hold also for Dsum-automata. Therefore, for the four measures that we consider, i.e. for $V \in\{$ Sum, Avg, Ratio, Dsum $\}$, deterministic $V$-automata are strictly less expressive than functional $V$-automata which are again strictly less expressive than the full class of $V$-automata.

The following result shows that unambiguous $V$-automata are as expressive as functional $V$-automata, modulo an exponential blow-up. This blow-up is worst-case unavoidable, already for non-deterministic and unambiguous finite state automata [33.

Lemma 2.3. Let $V \in\{$ Sum, Avg, Dsum, Ratio $\}$. For all functional $V$-automaton with $n$ states we can construct an equivalent unambiguous $V$-automaton with $O\left(n \cdot 2^{n}\right)$ states.

Proof. Our proof is independent on the measure. Let $A=\left(Q, q_{I}, F, \delta, \gamma\right)$ be a functional $V$-automaton. We order the transitions of $\delta$ by a total order denoted by $<_{\delta}$. We construct an unambiguous automaton $A^{\prime}=\left(Q^{\prime}, q_{I}^{\prime}, F^{\prime}, \delta^{\prime}, \gamma^{\prime}\right)$ equivalent to $A$, where:

- $Q^{\prime}=Q \times 2^{Q}$;

- $q_{i}^{\prime}=\left(q_{I}, \varnothing\right)$;

- $F^{\prime}=F \times\{P \subseteq Q \mid F \cap P=\varnothing\}$;

- $\gamma^{\prime}:\left((p, P), a,\left(p^{\prime}, P^{\prime}\right)\right) \mapsto \gamma\left(p, a, p^{\prime}\right)$;

Before defining $\delta^{\prime}$ formally, let us explain intuitively the semantics of the states in $Q^{\prime}$. The automaton $A^{\prime}$ will guess a run of $A$ on first state component (called the current run). A pair $(p, P)$ represents the state $p$ of current run in the original automaton $A$ while $P$ represents the states reached by all the runs that are greater than the current run (w.r.t. the order $<_{\delta}$ lexigraphically extended to runs). At the end of the word, the run is accepting iff $p$ is accepting and there is no accepting state in $P$. In other words, a run of $A^{\prime}$ on a word $w$ is accepting iff the run it defines on the first component is the biggest accepting run of $A$ on $w$.

When a new letter $a \in \Sigma$ is read, $A^{\prime}$ guesses a transition from $p$ to some state $p^{\prime}$, and goes to the state $\left(p^{\prime}, S_{P} \cup S_{p, a, p^{\prime}}\right)$, where $S_{P}$ are the successor states of of $P$ by $\delta$ on the input $a$, and $S_{p, a, p^{\prime}}$ are all the states reached from $p$ by a transition on $a$ bigger than $\left(p, q, p^{\prime}\right)$ (according to $<_{\delta}$ ).

Formally, $\left((p, P), a,\left(p^{\prime}, P^{\prime}\right)\right) \in \delta^{\prime}$ iff

- $\left(p, a, p^{\prime}\right) \in \delta$;

- $P^{\prime}=\left\{q^{\prime} \mid \exists q \in P,\left(q, a, q^{\prime}\right) \in \delta\right\} \cup\left\{p^{\prime \prime} \mid\left(p, a, p^{\prime \prime}\right) \in \delta \wedge\left(p, a, p^{\prime}\right)<_{\delta}\left(p, a, p^{\prime \prime}\right)\right\}$.

It is clear by construction that $A$ and $A^{\prime}$ define the same domain. As $A$ is functional, they also define the same function, because the value of a word is equal to the value of any run on it, and in particular to the value of the smallest run (w.r.t $<_{\delta}$ ). 
Group Automata Whenever possible, we generalise our result to group automata. Group automata are defined as weighted automata, except that the weights are taken from a group structure. Since there are no operation to combine the values of all the runs on the same input word, group automata define functions from words to set of values. Let us recall the definition of a group. A group is a structure $(W, \cdot \mathbb{1})$, where $W$ is a set, $\cdot: W \times W \rightarrow W$ is an associative operation, $\mathbb{1} \in W$ is a two sided identity element for - over $W$, and each element $s \in W$ admits an inverse $s^{-1} \in W$, such that $s^{-1} \cdot s=s \cdot s^{-1}=\mathbb{1}$ (the inverse is unique).

A group automaton over $\Sigma$ and a group $(W, \cdot, \mathbb{1})$ is a tuple $A=\left(Q, q_{I}, F, \delta, \gamma\right)$ where $Q, q_{I}, F, \delta$ are defined as for weighted automata, and $\gamma: \delta \rightarrow W$ is the weight function. Runs are defined as for weighted automata, and the value $V(\rho)$ of a run is obtained by taking the product of the weights of the transitions of $\rho$. The language defined by $A$, denoted by $L_{A}$, is a function from $\Sigma^{+}$to finite subsets of $W$, defined by $L_{A}(w)=\{V(\rho) \mid \rho$ is an accepting run on $w\}$.

All the notions defined for weighted automata, such as functionality, accessible states, etc. carry over to group automata. When a group automaton $A$ is functional, we rather write $L_{A}(w)=v$ instead of $L_{A}(w)=\{v\}$. Finally, we assume that the operations over group elements are computable in polynomial time in the size of their representation.

When considering only the relation induced by weighted $V$-automata, it turns out that they are equivalent to group automata, for $V \in\{$ Sum, Avg, Dsum $\}$.

Lemma 2.4. Let $V \in\{$ Sum, Avg, Dsum $\}$. There exists a group $G_{V}$ and a partial function $\psi: G_{V} \rightarrow \mathbb{Q}$, such that for all weighted $V$-automaton $A$, one can construct, in linear-time, a group automaton $A_{g}$ over $G_{V}$ such that for all $w \in \Sigma^{+}$, we have:

- for all $v \in \mathbb{Q}$, if $(w, v) \in R_{A}^{V}$, there exists $s \in G_{V}$ s.t. $s \in L_{A_{g}}(w)$ and $\psi(s)=v$, - for all $s \in G_{V}$ s.t. $s \in L_{A_{g}}(w),(w, \psi(s)) \in R_{A}^{V}$.

Proof. For Sum and Avg, we define $G_{\text {Sum }}=(\mathbb{Z},+, 0)$ and $G_{\text {Avg }}=\left(\mathbb{Z}^{2}, \cdot,(0,0)\right)$, where $\cdot$ is the pairwise sum. An Avg-automaton $A$ can be turned into a group automaton over $G_{\text {Avg }}$ by replacing each weight $v$ in $A$ with the pair $(v, 1)$. Then, it suffices to take $\psi$ defined by $\psi((v, n))=v / n$ for all $n \geq 1$, otherwise $\psi$ is undefined.

For Dsum-automata, consider the group $G_{\text {Dsum }}=(W, \cdot, \mathbb{1})$, where $W=\mathbb{Q} \times \mathbb{Q}^{+}$(where $\mathbb{Q}^{+}$is the set of strictly positive rational numbers $), \cdot$ is defined by $(a, x) \cdot(b, y)=\left(\frac{1}{y} a+b, x y\right)$, $\mathbb{1}=(0,1)$ is the identity element, and given $(a, x) \in W$, the inverse $(a, x)^{-1}$ is given by $(a, x)^{-1}=\left(-x a, x^{-1}\right)$.

Given $\lambda \in \mathbb{Q} \cap] 0,1[$, a Dsum-automaton $A$ on $\Sigma$ can be turned into a group automaton on $G_{\text {Dsum }}$, by replacing each weight $a$ in $A$ with the pair $(a, \lambda), a \in \mathbb{Z}$. Let $w=w_{0} \ldots w_{n} \in \Sigma$, and consider a run $\rho: q_{0} \stackrel{w}{\rightarrow} q_{n+1}$ on $w$ in $A$. Then, $\rho$ is valued by the pair $(a, x)=$ $\left(\frac{1}{\lambda^{n}} \gamma\left(q_{0}, w_{0}, q_{1}\right)+\cdots+\gamma\left(q_{n}, w_{n}, q_{n+1}\right), \lambda^{n+1}\right)$. Hence, $(a, x)$ codes the value $\psi((a, x))=$ $\frac{a x}{\lambda}=\operatorname{Dsum}(\rho)$.

As an immediate consequence of Lemma 2.4, we obtain that $A$ is functional iff $A_{g}$ is, and therefore, any procedure to decide functionality of group automata will allow one to decide functionality of weighted $V$-automata, for $V \in\{$ Sum, Avg, Dsum\}. We show such a procedure in the next section. We leave open the encoding of Ratio-automata as group automata. 


\section{Functionality Problem}

In this section, we consider the problem of deciding whether a weighted automaton is functional. In particular, Subsection 3.1 provides a general functionality test applying to group automata which, by Lemma 2.4, yields a procedure to test functionality of weighted $V$-automata, for $V \in\{$ Sum, Avg, Dsum $\}$. We also prove that functionality is decidable for Ratio-automata, by using different techniques.

3.1. Functionality of group automata. We start to introduce the notion of delay between two runs of a group automaton, which turns out to be the main ingredient of the functionality algorithm in Fig. 1,

Definition 3.1 (Delay). Let $G=(W, \cdot, \mathbb{1})$ be a group, and $A=\left(Q, q_{I}, F, \delta, \gamma\right)$ be a group automaton over $G$. Let $p, q \in Q$. A value $d \in W$ is a delay for $(p, q)$ if $A$ admits two runs $\rho: q_{I} \stackrel{w}{\rightarrow} p, \rho^{\prime}: q_{I} \stackrel{w}{\rightarrow} q$ on $w \in \Sigma^{*}$ s.t. $\operatorname{delay}\left(\rho, \rho^{\prime}\right)={ }_{\operatorname{def}}(V(\rho))^{-1} \cdot V\left(\rho^{\prime}\right)=d$.

The following lemma shows that at most one delay can be associated with co-accessible pairs of states in a functional group automaton. This is related to the uniqueness of inverse elements.

Lemma 3.2 (One Delay). Let $A=\left(Q, q_{I}, F, \delta, \gamma\right)$ be a functional group automaton. For all pairs of states $(p, q)$ : If $(p, q)$ is co-accessible, then $(p, q)$ admits at most one delay.

Proof. Let $(p, q)$ be a co-accessible pair of states. Assume that $(p, q)$ admits two delays $d_{1}, d_{2}$. We show that if $A$ is functional, then $d_{1}=d_{2}$. Let $\rho_{1}: q_{0} \stackrel{w_{1} \mid n_{1}}{\longrightarrow} p, \rho_{1}^{\prime}: q_{0} \stackrel{w_{1} \mid m_{1}}{\longrightarrow} q$ (resp. $\rho_{2}: q_{0} \stackrel{w_{2} \mid n_{2}}{\longrightarrow} p, \rho_{2}^{\prime}: q_{0} \stackrel{w_{2} \mid m_{2}}{\longrightarrow} q$ ) be a run witnessing the delay $d_{1}=\operatorname{delay}\left(\rho_{1}, \rho_{1}^{\prime}\right)$ (resp. $d_{2}=\operatorname{delay}\left(\rho_{2}, \rho_{2}^{\prime}\right)$ ). Since $(p, q)$ is co-accessible, there exists a word $u$ and two runs $\rho_{3}: p \stackrel{u \mid l}{\longrightarrow} f \in F, \rho_{3}^{\prime}: q \stackrel{u \mid s}{\longrightarrow} f^{\prime} \in F$. The hypothesis of functionality yields:

$$
\begin{aligned}
& m_{1} \cdot s=n_{1} \cdot l \Rightarrow n_{1}^{-1} \cdot m_{1}=l \cdot s^{-1} \\
& n_{2} \cdot l=m_{2} \cdot s \Rightarrow l \cdot s^{-1}=n_{2}^{-1} \cdot m_{2}
\end{aligned}
$$

From the above equations, we get:

$$
n_{1}^{-1} \cdot m_{1}=l \cdot s^{-1}=n_{2}^{-1} \cdot m_{2} \Rightarrow m_{1}^{-1} \cdot n_{1}=m_{2}^{-1} \cdot n_{2}
$$

We are now ready to define an algorithm (Algorithm 1) that checks the functionality of a group automaton over a group $(W, \cdot, \mathbb{1})$. In a first step, such a procedure computes all coaccessible pairs of states. Then, it explores the set of accessible pairs of states in a forward manner and computes the delays associated with those pairs. If two different delays are associated with the same pair, or if a pair of final states with a delay different from $\mathbb{1}$ (the neutral element of the group) is reached, the test stops and returns that the automaton is not functional (by Lemma 3.2 and by definition of functionality). Otherwise, it goes on until all co-accessible (and accessible) pairs have been visited and concludes that the automaton is functional.

If the algorithm returns No, it is either because a pair of accepting states with a delay different from 1 has been reached, which gives a counter-example to functionality, or because a pair of states with different delays has been found, so $A$ is not functional by Lemma 3.2 . 


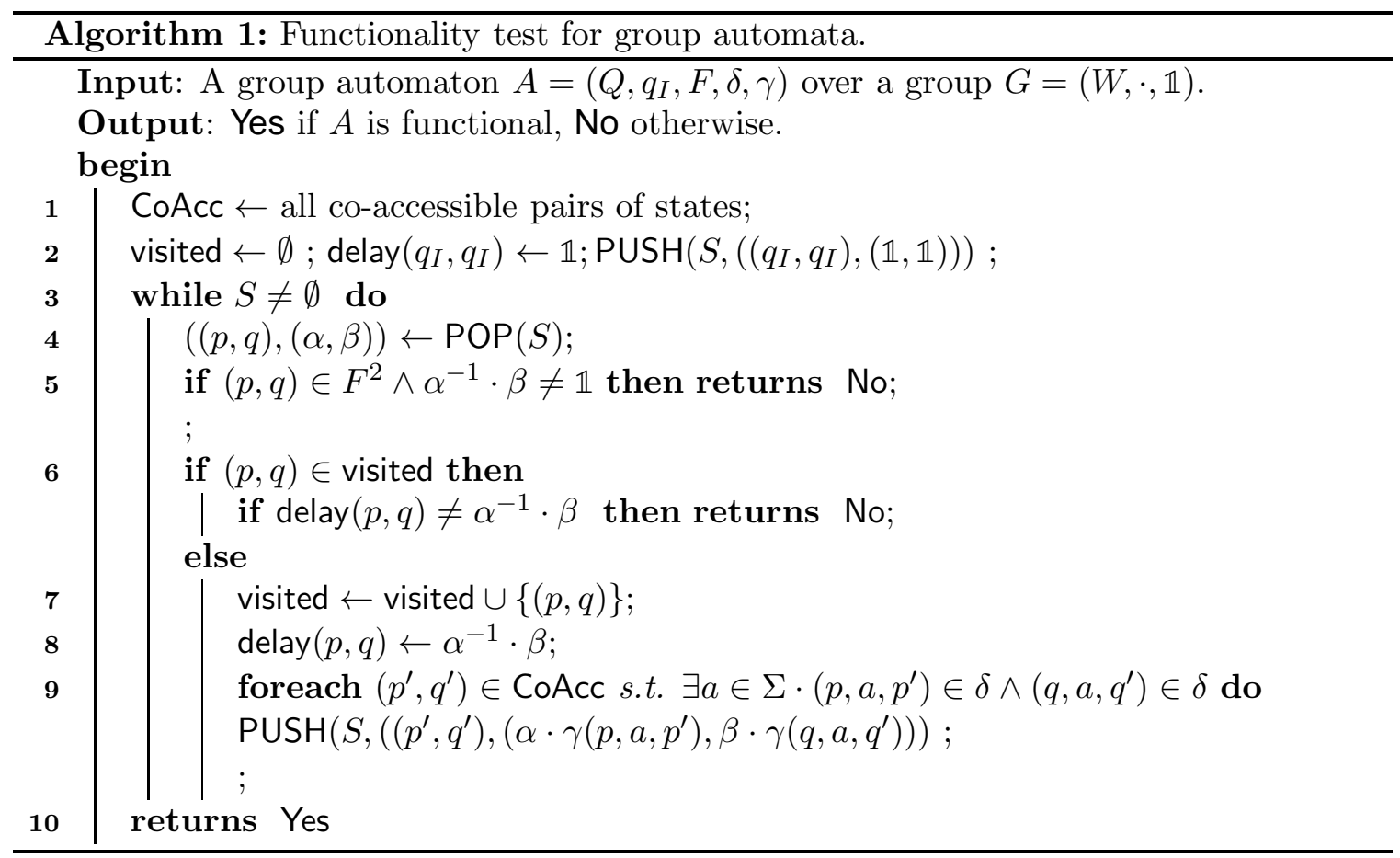

Lemma 3.4 provides a pumping argument useful to establish the converse. In detail, given a non functional automaton $A$, Lemma 3.4 establishes that $A$ admits two accepting runs witnessing non-functionality (i.e. on the same word $w$ and with different values) for which any pair of states that repeats twice has two different delays. The proof of Lemma 3.4 relies on the following key result:

Lemma 3.3. Let $A=\left(Q, q_{I}, F, \delta, \gamma\right)$ be a group automaton. Let $w_{1}, w_{2}, w_{3} \in \Sigma^{*}$ such that there exist $p, p^{\prime}, q, q^{\prime} \in Q$ and the following runs:

$$
\begin{array}{lll}
\rho_{1}: q_{I} \stackrel{w_{1} \mid n_{1}}{\longrightarrow} p & \rho_{2}: p \stackrel{w_{2} \mid n_{2}}{\longrightarrow} p & \rho_{3}: p \stackrel{w_{3} \mid n_{3}}{\longrightarrow} q \\
\rho_{1}^{\prime}: q_{I} \stackrel{w_{1} \mid m_{1}}{\longrightarrow} p^{\prime} & \rho_{2}: p^{\prime} \stackrel{w_{2} \mid m_{2}}{\longrightarrow} p^{\prime} & \rho_{3}: p^{\prime} \stackrel{w_{3} \mid m_{3}}{\longrightarrow} q^{\prime}
\end{array}
$$

s. t. delay $\left(\rho_{1}, \rho_{1}^{\prime}\right)=\operatorname{delay}\left(\rho_{1} \rho_{2}, \rho_{1}^{\prime} \rho_{2}^{\prime}\right)$. Then, we have delay $\left(\rho_{1} \rho_{2} \rho_{3}, \rho_{1}^{\prime} \rho_{2}^{\prime} \rho_{3}^{\prime}\right)=\operatorname{delay}\left(\rho_{1} \rho_{3}, \rho_{1}^{\prime} \rho_{3}^{\prime}\right)$.

Proof. By hypothesis, we have:

$$
n_{1}^{-1} \cdot m_{1}=\left(n_{1} \cdot n_{2}\right)^{-1} \cdot m_{1} \cdot m_{2} \Rightarrow n_{1}^{-1} \cdot m_{1}=n_{2}^{-1} \cdot n_{1}^{-1} \cdot m_{1} \cdot m_{2}
$$

Therefore, $n_{3}^{-1} \cdot n_{1}^{-1} \cdot m_{1} \cdot m_{3}=n_{3}^{-1} \cdot n_{2}^{-1} \cdot n_{1}^{-1} \cdot m_{1} \cdot m_{2} \cdot m_{3}$, that implies $\left(n_{1} \cdot n_{3}\right)^{-1} \cdot\left(m_{1} \cdot m_{3}\right)=$ $\left(n_{1} \cdot n_{2} \cdot n_{3}\right)^{-1} \cdot m_{1} \cdot m_{2} \cdot m_{3}$ i.e. delay $\left(\rho_{1} \rho_{2} \rho_{3}, \rho_{1}^{\prime} \rho_{2}^{\prime} \rho_{3}^{\prime}\right)=\operatorname{delay}\left(\rho_{1} \rho_{3}, \rho_{1}^{\prime} \rho_{3}^{\prime}\right)$.

Lemma 3.4. Let $A=\left(Q, q_{I}, F, \delta, \gamma\right)$ be a group automaton. If $A$ is not functional, there exists a word $w=\sigma_{0} \ldots \sigma_{n}$ and two accepting runs on it, $\rho=q_{0} \sigma_{0} \ldots q_{n+1}, \rho^{\prime}=q_{0}^{\prime} \sigma_{0} \ldots q_{n+1}^{\prime}$, such that $V(\rho) \neq V\left(\rho^{\prime}\right)$ and for all positions $i<j$ in $w$, if $\left(q_{i}, q_{i}^{\prime}\right)=\left(q_{j}, q_{j}^{\prime}\right)$ then $\operatorname{delay}\left(\rho_{i}, \rho_{i}^{\prime}\right) \neq \operatorname{delay}\left(\rho_{j}, \rho_{j}^{\prime}\right)$.

Proof. Let $w=\sigma_{0} \ldots \sigma_{n}, w \in \operatorname{dom}(A)$ such that $\left|R_{A}(w)\right|>1$. Clearly, there exist two runs $\rho=q_{0} \sigma_{0} \ldots q_{n+1}, \rho^{\prime}=q_{0}^{\prime} \sigma_{0} \ldots q_{n+1}^{\prime}$ on $w$ such that $V(\rho) \neq V\left(\rho^{\prime}\right)$. This implies delay $\left(\rho, \rho^{\prime}\right) \neq 1$. Suppose that there are two positions $i<j$ such that $\left(q_{i}, q_{i}^{\prime}\right)=\left(q_{j}, q_{j}^{\prime}\right)$ and $\operatorname{delay}\left(\rho_{i}, \rho_{i}^{\prime}\right)=$ delay $\left(\rho_{j}, \rho_{j}^{\prime}\right)$. Then, Lemma 3.3 applies and we can shorten the runs $\rho, \rho^{\prime}$ by removing the 
slices starting from position $i$ and ending in position $j$. In particular, we obtain two new runs $\bar{\rho}=q_{0} \sigma_{0} \ldots q_{i} \sigma_{j+1} \ldots q_{n+1}, \bar{\rho}^{\prime}=q_{0}^{\prime} \sigma_{0} \ldots q_{i} \sigma_{j+1} \ldots q_{n+1}^{\prime}$ on $\bar{w}=\sigma_{0} \ldots \sigma_{i-1} \sigma_{j+1} \ldots \sigma_{n}$ with the following property: $\operatorname{delay}\left(\bar{\rho}, \bar{\rho}^{\prime}\right)=\operatorname{delay}\left(\rho, \rho^{\prime}\right) \neq 1$. Iterating the above procedure we recover a pair of runs $\left(\widehat{\rho}, \widehat{\rho}^{\prime}\right)$ on $\widehat{w}$ such that delay $\left(\widehat{\rho}, \widehat{\rho}^{\prime}\right) \neq \mathbb{1}$ and for all positions $i<j$ in $\widehat{w}$, if $\left(q_{i}, q_{i}^{\prime}\right)=\left(q_{j}, q_{j}^{\prime}\right)$ then $\operatorname{delay}\left(\widehat{\rho}_{i}, \widehat{\rho}_{i}^{\prime}\right) \neq \operatorname{delay}\left(\widehat{\rho}_{j}, \widehat{\rho}_{j}^{\prime}\right)$, fulfilling the goals of Lemma 3.4

If there are two runs witnessing non-functionality without repetitions of pairs of states, the algorithm can find a pair of final states with a delay different from $\mathbb{1}$. Otherwise the algorithm will return No at line 6, if not before. Therefore we get:

Theorem 3.5. Let $A=\left(Q, q_{I}, F, \delta, \gamma\right)$ be a group automaton. Algorithm 1 returns Yes on $A$ iff $A$ is functional and terminates within $O\left(|A|^{2}\right)$ steps.

Proof. The procedure explores the co-accessible part of $A \times A$ in a depth-first-search manner. In particular, unless a condition for early termination (answering No) is encountered, each edge of the (co-accessible and accessible part of) $A \times A$ is visited once.

Given the above premise, assume that $A$ is not functional. Then by Lemma 3.4, $A$ admits two runs witnessing non functionality for which any pair of states that repeat twice have different delays. Thus, the exploration of such a pair of parallel runs in Algorithm 1 will eventually stop the procedure returning No, as soon as either a pair of co-accessible states is visited twice with different delays, or the final states are reached with a delay different from $\mathbb{1}$. Conversely, assume that the algorithm returns No. Then, this happens either at line 5 or at line 6 . In the first case, the algorithm exhibits exactly a pair of runs witnessing non-functionality (by definition). In the second case, the answer is correct by Lemma 3.2.

The complexity follows from the following observations. At line 1 the set of co-accessible pairs of states can be obtained in $O\left(|A|^{2}\right)$ steps by proceeding as follows. First, compute $A \times A$ and reverse its edges. Then for each pair $\left(q, q^{\prime}\right), q \in F, q^{\prime} \in F$, mark it as co-accessible and use it to discover new co-accessible pairs of states via a depth-first-search visit on (nonmarked) states in $A \times A$. Finally, within the main loop at line 3 each co-accessible pair of states is inserted into $S$ (with a corresponding delay) at most once.

Remark 3.6. Functionality of Sum-automata have been shown decidable in [23]. Our functionality algorithm on weighted automata over groups specialized for Sum-automata corresponds to the functionality algorithm for Sum-automata defined in [23]. Algorithm 11 can also be applied to word transducers for which functionality has been shown to be decidable in PTIME with similar techniques in [4].

Corollary 3.7 follows immediately from Lemma 2.4 and Theorem 3.5.

Corollary 3.7. The functionality problem is decidable in PTime for $V$-automata, $V \in$ $\{$ Sum, Avg, Dsum\}.

Remark 3.8. We remark that our functionality test could be applied also to a more general framework, where the weight-set $W$ of the considered group automaton is equipped with an equivalence relation $\sim_{W}$, and two runs are considered equivalent iff the corresponding values are equivalent w.r.t. $\sim_{W}$. In particular, $\sim_{W}$ needs to fulfill the following properties in order to be able to show uniqueness of the delay (modulo $\sim_{W}$ ) and termination of the functionality test: (1) it is a congruence, i.e. $\forall a, b, c, d \in W$ if $a \sim_{W} b$ and $c \sim_{W} d$, then $a \cdot c \sim_{W} b \cdot d ;(2)$ for all $a, b, c \in W$ if $a \nsim_{W} b$ then $a \cdot c \nsim_{W} b \cdot c$. 
3.2. Functionality of Ratio-automata. Unlike Sum, Avg and Dsum-automata, it is unclear whether Ratio-automata can be encoded in term of group automata. Intuitively, to provide such an encoding we would assign to each edge a pair of natural numbers, where the first component is the edge-reward and the second component is the edge-cost. Thus, each run $\rho$ is assigned the value $(n, m)$, where $n$ (resp. $m$ ) is the sum of the rewards (resp. costs) along the run, and two runs $\rho, \rho^{\prime}$ with values $(n, m),\left(n^{\prime}, m^{\prime}\right)$ need to be considered equivalent iff $n m^{\prime}=n^{\prime} m$. Unfortunately, the induced equivalence relation (where $(n, m)$ is equivalent to $\left(n^{\prime}, m^{\prime}\right)$ iff $\left.n m^{\prime}=n^{\prime} m\right)$ is not a congruence. Therefore, the results developed in the previous subsection do not apply to this class of weighted automata (at least to this encoding) as the quotient of the set of pairs by this equivalence relation is not a group (cf. Remark (3.8). In fact, it is still open whether there exists a good notion of delay for Ratioautomata that would allow us to design an efficient algorithm to test functionality. However deciding functionality can be done by using a short witness property of non-functionality, as shown in the following lemma.

Lemma 3.9 (Pumping). Let $A$ be a Ratio-automaton with $n$ states. $A$ is not functional iff there exist a word $w$ such that $|w|<4 n^{2}$ and two accepting runs $\rho, \rho^{\prime}$ on $w$ such that $\operatorname{Ratio}(\rho) \neq \operatorname{Ratio}\left(\rho^{\prime}\right)$.

Proof. We prove the existence of a short witness for non-functionality. The other direction is obvious. Let $w$ be a word such that $|w| \geq 4 n^{2}$ and there exist two accepting runs $\rho_{1}, \rho_{2}$ on $w$ such that $\operatorname{Ratio}(\rho) \neq \operatorname{Ratio}\left(\rho^{\prime}\right)$. Since $|w| \geq 4 n^{2}$, there exist states $p, q \in Q, p_{f}, q_{f} \in F$ and words $w_{0}, w_{1}, w_{2}, w_{3}, w_{4}$ such that $w=w_{0} w_{1} w_{2} w_{3} w_{4}$ and $\rho, \rho^{\prime}$ can be decomposed as follows:

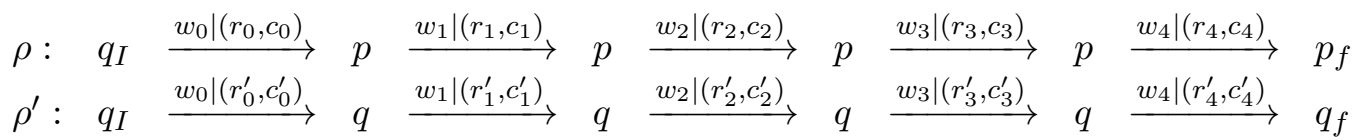

where $r_{i}, c_{i}$ denotes the sum of the rewards and the costs respectively on the subruns of $\rho$ on $w_{i}$, and similarly for $r_{i}^{\prime}, c_{i}^{\prime}$.

By hypothesis we know that $\left(\sum_{i=0}^{4} r_{i}\right) \cdot\left(\sum_{i=0}^{4} c_{i}^{\prime}\right) \neq\left(\sum_{i=0}^{4} c_{i}\right) \cdot\left(\sum_{i=0}^{4} r_{i}^{\prime}\right)$. For all subsets $X \subseteq\{1,2,3\}$, we denote by $w_{X}$ the word $w_{0} w_{i_{1}} \ldots w_{i_{k}} w_{4}$ if $X=\left\{i_{1}<\cdots<i_{k}\right\}$. For instance, $w_{\{1,2,3\}}=w, w_{\{1\}}=w_{0} w_{1} w_{4}$ and $w_{\{\}}=w_{0} w_{4}$. Similarly, we denote by $\rho_{X}, \rho_{X}^{\prime}$ the corresponding runs on $w_{X}$. We will show that there exists $X \subsetneq\{1,2,3\}$ such that $\operatorname{Ratio}\left(\rho_{X}\right) \neq \operatorname{Ratio}\left(\rho_{X}^{\prime}\right)$. Suppose that for all $X \subsetneq\{1,2,3\}$, we have $\operatorname{Ratio}\left(\rho_{X}\right)=\operatorname{Ratio}\left(\rho_{X}^{\prime}\right)$. We now show that it implies that $\operatorname{Ratio}(\rho)=\operatorname{Ratio}\left(\rho^{\prime}\right)$, which contradicts the hypothesis. For all $X \subseteq\{1,2,3\}$, we let:

$$
L_{X}=\left(\sum_{i \in X \cup\{0,4\}} r_{i}\right) \cdot\left(\sum_{i \in X \cup\{0,4\}} c_{i}^{\prime}\right) \quad R_{X}=\left(\sum_{i \in X \cup\{0,4\}} c_{i}\right) \cdot\left(\sum_{i \in X \cup\{0,4\}} r_{i}^{\prime}\right)
$$

By hypothesis, $L_{\{1,2,3\}} \neq R_{\{1,2,3\}}$ and for all $X \subsetneq\{1,2,3\}, L_{X}=R_{X}$. The following equalities can be easily verified:

$$
\begin{aligned}
& L_{\{\}}+L_{\{1,2\}}+L_{\{1,3\}}+L_{\{2,3\}}-L_{\{1\}}-L_{\{2\}}-L_{\{3\}}=L_{\{1,2,3\}} \\
& R_{\{\}}+R_{\{1,2\}}+R_{\{1,3\}}+R_{\{2,3\}}-R_{\{1\}}-R_{\{2\}}-R_{\{3\}}=R_{\{1,2,3\}}
\end{aligned}
$$

Then, since by hypothesis we have $L_{X}=R_{X}$ for all $X \subsetneq\{1,2,3\}$, we get $L_{\{1,2,3\}}=R_{\{1,2,3\}}$, which is a contradiction. Thus there exists $X \subsetneq\{1,2,3\}$ such that $L_{X} \neq R_{X}$. In other words, there exists $X \subsetneq\{1,2,3\}$ such that $\operatorname{Ratio}\left(\rho_{X}\right) \neq \operatorname{Ratio}\left(\rho_{X}^{\prime}\right)$. This shows that when a witness of non-functionality has length at least $4 n^{2}$, we can find a strictly smaller witness of functionality. 
Theorem 3.10. Functionality is decidable in CoNP for Ratio-automata, and in PTime if the rewards and costs are unary encoded.

Proof. As a consequence of Lemma 3.9, we can design an NP procedure that will check nonfunctionality by guessing runs of length at most $4 n^{2}$ and by testing, in polynomial time, that they have different ratio values, where $n$ is the number of states.

To get the PTime upper bound, we design a CoNLogSpace algorithm as follows. The algorithm guesses two runs $\rho_{1}$ and $\rho_{2}$ of the Ratio-automaton $A$ on the same input, and stores in memory a counter value $c$, the two states $q_{1}$ and $q_{2}$ reached so far by the runs, and the current sums $R_{1}, C_{1}, R_{2}, C_{2}$ of costs and rewards of the two runs respectively. At each step, it guesses a new symbol $\sigma \in \Sigma$, two transitions $q_{1} \stackrel{\sigma \mid\left(r_{1}, c_{1}\right)}{\longrightarrow} q_{1}^{\prime}$ and $q_{2} \stackrel{\sigma \mid\left(r_{2}, c_{2}\right)}{\longrightarrow} q_{2}^{\prime}$ of $A$, and updates its memory to $\left(c+1, q_{1}^{\prime}, q_{2}^{\prime}, R_{1}+r_{1}, C_{1}+c_{1}, R_{2}+r_{2}, C_{2}+c_{2}\right)$. The algorithm stops in a configuration $\left(c, q_{1}, q_{2}, R_{1}, C_{1}, R_{2}, C_{2}\right)$ with negative answer whenever $c=4 n^{2}+1$, and with a positive answer whenever $c \leq 4 n^{2}, q_{1}, q_{2}$ are accepting, and $R_{1} C_{2} \neq R_{2} C_{1}$.

The correctness of this algorithm directly follows from Lemma 3.9, Let us show that each configuration uses only a logarithmic space to be stored. Let $M$ be the largest value (among rewards and costs) that occurs on the transitions of $A$. Let $|M|$ denote the size of $M$. Since rewards and costs are encoded in unary, we have $|M|=M$. Now, the algorithm first converts the rewards and costs in binary (in logspace), and then, each configuration $\left(c, q_{1}, q_{2}, R_{1}, C_{1}, R_{2}, C_{2}\right)$ takes only logarithmic space, because for any $\alpha \in\left\{R_{1}, C_{1}, R_{2}, C_{2}\right\}$, $\alpha \leq 4 n^{2} M=4 n^{2}|M|$, and therefore $\log (\alpha) \leq 2 \log (2 n)+\log (|M|)$. We can conclude since CoNLogSpace $\subseteq$ PTime.

Remark 3.11. The pumping result in Lemma 3.9 states that if some Ratio-automaton with $n$ states is not functional, there exists a witness of non-functionality whose length is bounded by $4 n^{2}$, where $n$ is the number of states. Such a property also holds for Dsumautomata (and is well-known for Sum and Avg-automata), but with the smaller bound $3 n^{2}$. Those bounds are used to state the existence of two runs on the same word such that the same pair of states is repeated 3 or 4 times along the two runs. Then it is proved that one can remove some part in between two repetitions and get a smaller word with two different output values. However for Ratio-automata, three repetitions are not enough to be able to shorten non-functionality witnesses. For instance, consider the following two runs on the alphabet $\{a, b, c, d\}$ and states $\left\{q_{I}, p, q, p_{f}, q_{f}\right\}$ where $p_{f}, q_{f}$ are final (those two runs can easily be realized by some Ratio-automaton):

$$
\begin{aligned}
& \rho: \quad q_{I} \stackrel{a \mid(2,2)}{\longrightarrow} \quad p \stackrel{b \mid(1,2)}{\underset{b \mid(2,1)}{\longrightarrow}} p \stackrel{c \underset{c \mid(1,1)}{\longrightarrow}}{\stackrel{c \mid(2,2)}{\longrightarrow}} p \stackrel{d \mid(1,1)}{\longrightarrow} p_{f} \\
& \rho^{\prime}: \quad q_{I} \stackrel{a \mid(1,2)}{\longrightarrow} q \stackrel{b \mid(2,1)}{\longrightarrow} q \stackrel{c \mid(1,1)}{\longrightarrow} q \stackrel{d \mid(2,1)}{\longrightarrow} q_{f}
\end{aligned}
$$

It is easy to verify that the word abcd has two outputs given by $\rho$ and $\rho^{\prime}$ while the words $a d, a b d$ and $a c d$ has one output. For instance, the two runs $q_{I} \stackrel{a \mid(2,2)}{\longrightarrow} p \stackrel{d \mid(1,1)}{\longrightarrow} p_{f}$ and $q_{I} \stackrel{a \mid(1,2)}{\longrightarrow} q \stackrel{d \mid(2,1)}{\longrightarrow} q_{f}$ on $a d$ have both value 1 .

\section{Verification Problems}

In this section, we investigate several decision problems for functional $V$-automata as defined in [11], $V \in\{$ Sum, Avg, Dsum, Ratio $\}$. Given two $V$-automata $A, B$ over $\Sigma$ (and with the 
same discount factor when $V=$ Dsum) and a threshold $\nu \in \mathbb{Q}$, we define the following decision problems, where $\triangleright \in\{>, \geq\}$ :

$\begin{array}{lll}\triangleright \nu \text {-Emptiness } & L_{A}^{\triangleright \nu} \neq \varnothing \quad \text { holds if there exists } w \in \Sigma^{+} \text {such that } L_{A}(w) \triangleright \nu \\ \triangleright \nu \text {-Universality } & L_{A} \triangleright \nu & \text { holds if for all } w \in \operatorname{dom}(A), L_{A}(w) \triangleright \nu . \\ \text { Inclusion } & L_{A} \leq L_{B} \quad \text { holds if for all } w \in \Sigma^{+}, L_{A}(w) \leq L_{B}(w) \\ \text { Equivalence } & L_{A}=L_{B} \quad \text { holds if for all } w \in \Sigma^{+}, L_{A}(w)=L_{B}(w)\end{array}$

Theorem 4.1 (resp. Theorem 4.2) below proves that the $\triangleright \nu$-emptiness (resp. universality) problem can be solved in polynomial time for functional Sum-, Avg-, and Ratio-automata. As for functional Dsum-automata, we provide a PTime upper bound for the $>\nu$-emptiness (resp. $\geq \nu$-universality) problem. The $\geq \nu$-emptiness (resp. $>\nu$-universality) on functional Dsum-automata has been recently shown in PSpace in [9], as an aside result of a partial solution 3 to the notorious target discounted-sum problem. The latter is known to be connected to various areas and open problems in mathematics and computer science, such as e.g. the universality of (nondeterministic) Dsum-automata, Dsum-games with imperfect information or multi-objectives [10, 12, as well as piecewise affine maps and generalizations of the Cantor set [9].

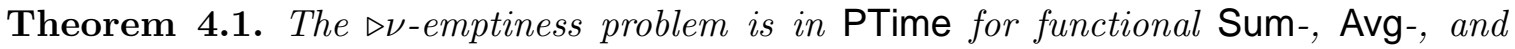
Ratio-automata. The $>\nu$-emptiness (resp. $\geq \nu$-emptiness) problem is in PTime (resp. PSpace [9]) for functional Dsum-automata.

Proof. For Sum-automata, let $A=\left(Q, q_{I}, F, \delta, \gamma\right)$ be a Sum-automaton. Wlog we assume that all states of $A$ are both accessible from an initial state and co-accessible from a final state (such property can be ensured via a PTime transformation [30]). First, $L_{A}^{\triangleright \nu} \neq \varnothing$ if $A$ contains a strictly positive cycle, otherwise one inverts the weights and computes a shortest path from an initial to a final state. If the sum $\beta$ of such a path satisfies $-\nu \triangleright \beta$ then the language is non-empty. Both steps are handled by the classical Bellman-Ford algorithm.

For Avg-automata, let $A=\left(Q, q_{I}, F, \delta, \gamma\right)$ be an Avg-automaton. We can assume $\nu=0$ since the $\triangleright \nu$-emptiness problem for Avg-automata reduces to the $\triangleright 0$-emptiness problem for Sum-automata, by simply reweighting the input automaton. Then, $L_{A}^{\triangleright 0} \neq \emptyset$ iff $A$ admits a path to a final state whose sum of the weights is $\triangleright 0$, that can be easily checked in PTime.

For Dsum automata, let $A=\left(Q, q_{I}, F, \delta, \gamma\right)$ be a Dsum-automaton. We show that $L_{A}^{>\nu} \neq \emptyset$ iff Player 0 has a strategy to ensure a play from $v_{I}$ with discounted sum greater than $\nu$ in the one player (infinite) Dsum game $\Gamma=\left(V, E, w,\left\langle V_{0}, V_{1}\right\rangle\right)$, where:

- $V=\left\{p \mid p \in Q \wedge \exists w \in \Sigma^{*}(p \stackrel{w}{\rightsquigarrow} f \in F)\right\}$

- $V_{0}=V, V_{1}=\emptyset$

- $E=(V \times(\Sigma \cup\{\zeta\}) \times V) \cap\left(\left\{\left(p, a, p^{\prime}\right) \mid\left(p, a, p^{\prime}\right) \in \delta\right\} \cup\{(p, \zeta, p) \mid p \in F\}\right)$, where $\zeta \notin \Sigma$ is a fresh symbol

- For each $e=\left(p, a, p^{\prime}\right) \in E$ : If $\left(p, a, p^{\prime}\right) \in \delta$, then $w(e)=\gamma\left(p, a, p^{\prime}\right)$, else $w(e)=0$.

Once proved the above equivalence, our complexity bound follows easily, since checking whether $L_{A}^{>\nu} \neq \emptyset$ reduces to solving a 1 player Dsum game (that is in PTime [3]).

If $L_{A}^{>\nu} \neq \emptyset$, then $A$ admits an accepting run $\rho$ such that $\operatorname{Dsum}(\gamma(\rho))>\nu$. By construction, $\Gamma$ admits an (infinite) path with a positive discounted sum, i.e. Player 0 has a (memoryless) strategy to win the one-player discounted sum game $\Gamma$.

\footnotetext{
${ }^{3}$ In particular, 9] provides a solution to the finite version of the target discounted-sum problem.
} 
Conversely, suppose that Player 0 has a strategy to win the one-player discounted sum game $\Gamma$. Let $r$ be an infinite path on $\Gamma$ consistent with a winning strategy for player 0 . Then $\operatorname{Dsum}(r)>0$. Let $W$ be the maximum absolute weight in $\Gamma$. For each prefix $r_{i}$ of length $i$ of $r$ we have:

$$
\begin{aligned}
& \operatorname{Dsum}\left(r_{i}\right)+\frac{W \lambda^{i}}{1-\lambda} \geq \operatorname{Dsum}(r) \Rightarrow \\
& \operatorname{Dsum}\left(r_{i}\right) \geq \operatorname{Dsum}(r)-\frac{W \lambda^{i}}{1-\lambda}
\end{aligned}
$$

Since $\operatorname{Dsum}(r)>\nu$, there exists $i^{*}$ such that $\operatorname{Dsum}(r)-\frac{W \lambda^{i^{*}}}{1-\lambda}>\nu$ that implies $\operatorname{Dsum}\left(r_{i^{*}}\right)>$ $\nu$. By construction, each path in $\Gamma$ can be extended to reach a node in $F$. Let $r_{i}^{\prime}=$ $r_{0}^{\prime} \ldots r_{m}^{\prime} \in F$ be such a continuation of $r^{i^{*}}$. By Equation 4.1, our choice of $i^{*}$ guarantees that $\operatorname{Dsum}\left(r_{i}^{\prime}\right)>\nu$. Since $A$ is functional, $r^{\prime}$ witnesses the existence of a word $w$ such that $L_{A}(w)>\nu$.

Finally, let $A$ be a Ratio-automaton, let $\nu=m / n$. We consider the Sum automaton $A^{\prime}$, where each edge of $A$ having reward $r$ and cost $c$ is replaced by an edge of weight $r n-c m$. It can be easily proved that $L_{A}^{\triangleright \nu} \neq \emptyset$ iff $L_{A^{\prime}}^{\triangleright \nu} \neq \emptyset$.

Theorem 4.2. Let $\nu \in \mathbb{Q}$. The $\triangleright \nu$-universality problem is $\mathrm{PTime}$ for functional $V$-automata, $V \in\{$ Sum, Avg, Ratio $\}$. The $\geq \nu$-universality (resp. $>\nu$-universality) problem is PTime (resp. PSpace [9]) for functional Dsum-automata.

Proof. Let $A$ be a $V$-automaton, $V \in\{$ Sum, Avg, Dsum $\}$ and consider the $\geq \nu$-universality (resp. $>\nu$-universality) problem for $V$-automata. We check whether $A$ admits an accepting run with $V(\gamma(r))<\nu$ (resp. $V(\gamma(r)) \leq \nu$ ). This can be done in PTime for $V \in\{$ Sum, Avg, Ratio, Dsum $\}$ (resp. $V \in\{$ Sum, Avg, Ratio $\}$ ), with a procedure similar to the one applied in the proof of Theorem 4.1.

It is known that inclusion is undecidable for non-deterministic Sum-automata [25, 1], and therefore also for Avg and Ratio-automata. To the best of our knowledge, it is open whether it is decidable for Dsum-automata. This situation is strikingly different for functional automata as the inclusion problem is decidable for all the measures:

Theorem 4.3. Let $V \in\{$ Sum, Avg, Dsum, Ratio $\}$ and let $A, B$ be two $V$-automata where $B$ is functional. The inclusion problem $L_{A} \leq L_{B}$ is decidable. Moreover, if $V \in\{$ Sum, Avg, Dsum $\}$ then it is PSpace-c and if additionally $B$ is deterministic, it is in PTime.

Proof. Let $V \in\{$ Sum, Avg, Dsum $\}$. In a first step, we test the inclusion of the domains $\operatorname{dom}(A) \subseteq \operatorname{dom}(B)$ (it is well-known from theory of finite automata to be in PSpace-c and in PTime if $B$ is deterministic). Then we construct the product $A \times B$ as follows: $(p, q) \stackrel{a \mid n_{A}-n_{B}}{\longrightarrow}\left(p^{\prime}, q^{\prime}\right) \in \delta_{A \times B}$ iff $p \stackrel{a \mid n_{A}}{\longrightarrow} p^{\prime} \in \delta_{A}$ and $q \stackrel{a \mid n_{B}}{\longrightarrow} q^{\prime} \in \delta_{B}$. Then $L_{A} \not L_{B}$ iff $L_{A \times B}^{>0} \neq \varnothing$, which is decidable by Theorem 4.1 ,

Let $V=$ Ratio. As for the other measures we first check inclusion of the domains. We then define the product $A \times B$ of $A$ and $B$ as a labelled transition system whose set of transitions $\delta_{A \times B}$ is defined by $(p, q) \stackrel{a \mid\left(r_{1}, c_{1}, r_{2}, c_{2}\right)}{\longrightarrow}\left(p^{\prime}, q^{\prime}\right) \in \delta_{A \times B}$ iff $p \stackrel{a \mid\left(r_{1}, c_{1}\right)}{\longrightarrow} p^{\prime} \in \delta_{A}$ and $q \stackrel{a \mid\left(r_{2}, c_{2}\right)}{\longrightarrow} q^{\prime} \in \delta_{B}$. For all $t \in \delta_{A \times B}$, we let $r_{A}(t)$ be the reward of the transition $t$ projected on $A$. The values $c_{A}(t), r_{B}(t)$ and $c_{B}(t)$ are defined similarly. We let $\mathcal{P}(A \times B)$ be the Parikh image of the transitions of $A \times B$, i.e. the set of total functions $\alpha: \delta_{A \times B} \rightarrow \mathbb{N}$ such 
that there exist $w \in \Sigma^{+}$and a path labelled $w$ from the pair of initial states to a pair of accepting states, such that this path passes by $t$ exactly $\alpha(t)$ times, for all $t \in \delta_{A \times B}$. It is well-known by Parikh's theorem that $\mathcal{P}(A \times B)$ can be effectively represented by a set of linear constraints [31. We now define the set of vectors $\Gamma$ that are the Parikh images of accepting runs of $A \times B$ which, when projected on $A$, has a strictly bigger ratio value than the one obtained by the projection on $B$. In particular, the the set of vectors $\Gamma$ is given by:

$$
\left\{\alpha: \delta_{A \times B} \rightarrow \mathbb{N} \mid \alpha \in \mathcal{P}(A \times B), \frac{\sum_{t \in \delta_{A \times B}} \alpha(t) \cdot r_{A}(t)}{\sum_{t \in \delta_{A \times B}} \alpha(t) \cdot c_{A}(t)}>\frac{\sum_{t \in \delta_{A \times B}} \alpha(t) \cdot r_{B}(t)}{\sum_{t \in \delta_{A \times B}} \alpha(t) \cdot c_{B}(t)}\right\}
$$

It is easy to check that $\Gamma \neq \varnothing$ iff $L_{A} \not \leq L_{B}$. The set $\Gamma$ can be defined as the solutions over natural numbers of a system of equations in linear and quadratic forms (i.e. in which products of two variables are permitted). There is one variable $x_{t}$ for each $t \in \delta_{A \times B}$ that gives the number of times $t$ is fired in an accepting run of $A \times B$. It is decidable whether such a system has a solution [38, 20].

There is no known complexity bound for solving quadratic equations, so the proof above does not give us a complexity bound for the inclusion problem of functional Ratio-automata. However, thanks to the functionality test, which is in PSpace for Ratio-automata, we can test equivalence of two functional Ratio-automata $A_{1}$ and $A_{2}$ in PSpace:

Theorem 4.4. Let $V \in\{$ Sum, Avg, Dsum, Ratio $\}$. Equivalence of functional $V$-automata is PSpace-c.

Proof. The following algorithm can be used to test equivalence for all the considered measures: first check in PSpace that $\operatorname{dom}\left(A_{1}\right)=\operatorname{dom}\left(A_{2}\right)$ using the standard equivalence algorithm for non-deterministic finite automata. Then, check that the union of $A_{1}$ and $A_{2}$ is functional. The latter can be done in CoNP for Ratio-automata and in polynomial time for the other measures, using the functionality tests defined in the previous section (cf. Theorem 3.5, Corollary 3.7, and Theorem 3.10).

\section{Realizability Problem}

In this section, we consider the problem of quantitative language realizability. The realizability problem is better understood as a game between two players: the 'Player input' (the environment, also called Player $I$ ) and the 'Player output' (the controller, also called Player $O)$. Player $I$ (resp. Player $O$ ) controls the letters of a finite alphabet $\Sigma_{I}$ (resp. $\Sigma_{O}$ ). We assume that $\Sigma_{O} \cap \Sigma_{I}=\varnothing$ and that $\Sigma_{O}$ contains the special symbol $\dashv$ whose role is to stop the game. We let $\Sigma=\Sigma_{O} \cup \Sigma_{I}$.

Formally, the realizability game is a turn-based game played on an arena defined by a weighted automaton $A=\left(Q=Q_{O} \uplus Q_{I}, q_{I}, F, \delta, \gamma\right)$, whose set of states is partitioned into two sets $Q_{O}$ and $Q_{I}$, and such that $F \subseteq Q_{I}$ and $\delta \subseteq\left(Q_{O} \times \Sigma_{O} \times Q_{I}\right) \cup\left(Q_{I} \times \Sigma_{I} \times Q_{O}\right)$. Recall that by definition of the transitions of weighted automaton $\operatorname{dom}(A) \subseteq(\Sigma \backslash\{\dashv\})^{*} \dashv$. Player $I$ starts by giving an initial letter $i_{0} \in \Sigma_{I}$, Player $O$ responds providing a letter $o_{0} \in \Sigma_{O}$, then Player $I$ gives $i_{1}$ and Player $O$ responds $o_{1}$, and so on. Player $O$ has also the power to stop the game at any turn with the distinguishing symbol $\dashv$. In this case, the game results in a finite word $\left(i_{0} o_{0}\right)\left(i_{1} o_{1}\right) \ldots\left(i_{j} \dashv\right) \in \Sigma_{\dashv}^{+}$, otherwise the outcome of the game is an infinite word $\left(i_{0} o_{0}\right)\left(i_{1} o_{1}\right) \cdots \in(\Sigma \backslash\{-\})^{\omega}$. 
The players play according to strategies. A strategy for Player $O$ (resp. Player $I$ ) is a mapping $\lambda_{O}:\left(\Sigma_{I} \Sigma_{O}\right)^{*} \Sigma_{I} \rightarrow \Sigma_{O}$ (resp. $\left.\lambda_{I}:\left(\Sigma_{I} \Sigma_{O}\right)^{*} \rightarrow \Sigma_{I}\right)$. The outcome of the strategies $\lambda_{O}, \lambda_{I}$ is the word $w=i_{0} o_{0} i_{1} o_{1} \ldots$ denoted by outcome $\left(\lambda_{I}, \lambda_{O}\right)$ such that for all $0 \leq j \leq|w|$ (where $|w|=+\infty$ if $w$ is infinite), $o_{j}=\lambda_{O}\left(i_{0} \ldots i_{j-1}\right)$ and $i_{j}=\lambda\left(i_{0} \ldots o_{j}\right)$, and such that if $o_{j}=\dashv$ for some $j$, then $w=i_{0} \ldots o_{j}$. We denote by $\Lambda_{O}$ (resp. $\left.\Lambda_{I}\right)$ the set of strategies for Player $O$ (resp. Player $I$ ). A strategy $\lambda_{O} \in \Lambda_{O}$ is winning for Player $O$ if for all $\lambda_{I} \in \Lambda_{I}$, outcome $\left(\lambda_{I}, \lambda_{O}\right)$ is finite and $L_{A}$ (outcome $\left.\left(\lambda_{I}, \lambda_{O}\right)\right)>\nu$, where $\nu \geq 0$ a given threshold.

Given a weighted automaton $A$ and a threshold $\nu \geq 0$, the quantitative language realizability problem asks whether Player $O$ has a winning strategy and in that case, we say that $A$ is realizable. Our first result on realizability is negative: in particular, Subsection 5.1 shows that the realizability problem is undecidable for weighted functional Sum-, Avg-automata, and Ratio-automata. However, when deterministic weighted automata are considered, we can provide positive decidability results for all the considered measures (cfr. subsection 5.2).

5.1. Undecidability Results. We show that the halting problem for deterministic 2counter Minsky machines [28] can be reduced to the quantitative language realizability problem for (functional) Sum-automata (resp. Avg-automata). This entails our undecidability results w.r.t. realizability for $V$-automata $(V \in\{$ Sum,Avg,Ratio $\})$.

2-Counter Machines. A 2-counter machine $M$ consists of a finite set of control states $S$, an initial state $s_{I} \in S$, a final state $s_{F} \in Q$, a set $C$ of counters $(|C|=2)$ and a finite set $\delta_{M}$ of instructions manipulating two integer-valued counters. Instructions are of the form

$s: c:=c+1$ goto $s^{\prime}$

$s:$ if $c=0$ then goto $s^{\prime}$ else $c:=c-1$ goto $s^{\prime \prime}$.

Formally, instructions are tuples $\left(s, \alpha, c, s^{\prime}\right)$ where $s, s^{\prime} \in S$ are source and target states respectively, and the action $\alpha \in\{i n c, d e c, 0$ ? $\}$ applies to the counter $c \in C$. We assume that $M$ is deterministic: for every state $s \in S$, either there is exactly one instruction $(s, \alpha, \cdot, \cdot) \in \delta_{M}$ and $\alpha=i n c$, or there are two instructions $(s$, dec, $c, \cdot),(s, 0 ?, c, \cdot) \in \delta_{M}$.

A configuration of $M$ is a pair $(s, v)$ where $s \in S$ and $v: C \rightarrow \mathbb{N}$ is a valuation of the counters. An accepting run of $M$ is a finite sequence $\pi=\left(s_{0}, v_{0}\right) \delta_{0}\left(s_{1}, v_{1}\right) \delta_{1} \ldots \delta_{n-1}\left(s_{n}, v_{n}\right)$ where $\delta_{i}=\left(s_{i}, \alpha_{i}, c_{i}, s_{i+1}\right) \in \delta_{M}$ are instructions and $\left(s_{i}, v_{i}\right)$ are configurations of $M$ such that $s_{0}=s_{I}, v_{0}(c)=0$ for all $c \in C, s_{n}=s_{F}$, and for all $0 \leq i<n$, we have $v_{i+1}(c)=v_{i}(c)$ for $c \neq c_{i}$, and $(a)$ if $\alpha=$ inc, then $v_{i+1}\left(c_{i}\right)=v_{i}\left(c_{i}\right)+1(b)$ if $\alpha=\operatorname{dec}$, then $v_{i}\left(c_{i}\right) \neq 0$ and $v_{i+1}\left(c_{i}\right)=v_{i}\left(c_{i}\right)-1$, and $(c)$ if $\alpha=0$ ?, then $v_{i+1}\left(c_{i}\right)=v_{i}\left(c_{i}\right)=0$. The corresponding run trace of $\pi$ is the sequence of instructions $\bar{\pi}=\delta_{0} \delta_{1} \ldots \delta_{n-1}$. The halting problem is to decide, given a 2-counter machine $M$, whether $M$ has an accepting run. This problem is undecidable [28].

The encoding. We are now ready to present our encoding. The latter goes along the lines of the encodings in [15, 1] to prove undecidability results related to imperfect information games and weighted automata.

Given a deterministic 2-counter machine $M$, we construct a functional Sum-automaton $A=\left(Q, q_{I}, F, \delta, \gamma\right)$ (resp. Avg-automata), where $Q=Q_{O} \cup Q_{I} \cup F, \Sigma=\Sigma_{O} \cup \Sigma_{I}$ and $\delta \subseteq Q \times \Sigma \times Q$, such that $M$ halts if and only if $L(A)$ is realizable (with realizability threshold $\nu=0)$. In particular, $\Sigma_{O}=\delta_{M}$ and a strategy $\lambda_{O} \in \Lambda_{O}$ for Player $O$ is winning if and only 
if for each $\lambda_{I} \in \Lambda_{I}$, the projection of outcome $\left(\lambda_{I}, \lambda_{O}\right)$ onto $\Sigma_{O}$ is an accepting run of $M$. The alphabet $\Sigma_{I}$ for Player $I$ is the set of letters $\Sigma_{I}=\{g o\} \cup\left(\bigcup_{i=1,2}\{\right.$ cheatCit, cheatC $\left.i-\}\right) \cup$ $\left(\bigcup_{0 \leq j<|S|}\left\{\right.\right.$ cheatR: $\left.\left.s_{j}\right\}\right)$.

Intuitively, the role of Player $I$ is that of observing the play of Player $O$ and detecting whether he faithfully simulates $M$, or he cheats. If Player $O$ cheats by declaring the $i$-th counter equal to 0 when it is not (positive cheat), then Player $I$ can use the action cheatCi+, $i \in\{1,2\}$, to force all the runs but one (with weight $\leq 0$ ) to die. Similarly, if Player $O$ cheats by decrementing a counter with value zero (negative cheat) or on the structural properties of a run of $M$, then Player $I$ can win by playing the corresponding observing action : cheatCi-, for negative cheats on counter $i \in\{1,2\}$, or cheatR: $s_{j}$ for a cheat on the run through $M$ detected at state $s_{j}$.

In detail, the automaton $A$ consists of an initial state $q_{I}$ from which Player $I$ can nondeterministically jump to several gadgets: each gadget checks one of the properties of the sequence of actions provided by Player $O$, and verify whether Player $O$ simulates faithfully $M$ or he eventually cheats. More specifically, $q_{I}$ has one transition with weight 0 and label go to the set of gadgets listed below and described in detail in the rest of this subsection:

- two gadgets to observe positive cheats over a counter (one gadget for each counter $i \in$ $\{1,2\})$;

- two gadgets to observe negative cheats over a counter (one gadget for each counter $i \in$ $\{1,2\})$;

- a gadget to observe a structural cheat for each state $s \in S$ that can be traversed by a path in $M$;

- a neutral gadget, where Player $I$ simply observes the run provided by Player $O$ and let such a run to reach a final state as soon as Player $O$ provides an action simulating a step toward the halting state of $M$.

Due to the initial nondeterministic choice, each final state (in one of the gadget) is accessible throughout the evolution of the play and Player $O$ has to ensure that all the properties checked in the gadgets are fulfilled. Otherwise, Player $I$ will have the ability to use a letter in $\Sigma_{I} \backslash\{g o\}$ to let just one run (in the appropriate gadget) to survive, ensuring that such a run eventually reaches the final state with weight $\leq 0$.

We are now ready to present in detail the gadgets (cf. Figure 26). In particular, in each gadget the states belonging to $Q_{I}$ (resp. $Q_{O}$ ) are represented by a square node (resp. circle node), while final states are double lined 4 . Each transition in the gadgets leaving a node owned by Player $I$ is labelled by a pair of the form $(\sigma, w)$, where $\sigma \in \Sigma_{I}$ and $w \in\{-1,0,1\}$ is the weight of the transition. Symmetrically, each transition in the gadgets leaving a circle-node (i.e. a node owned by Player $O$ ) is labelled by a pair of the form $(\sigma, w)$, where $w \in\{-1,0,1\}$ is the weight and $\sigma \in \delta_{M}$ is an instruction of the counter machine. Namely, $\sigma$ is of the form $\left(s, \alpha, i, s^{\prime}\right)$, where $i \in\{1,2\}$ represents the counter, $\alpha \in\{$ inc, dec, 0 ? $\}$ and $s$ (resp. $s^{\prime}$ ) is the sourcing (resp. target) state. We use the notation - when a symbol within an instruction could be any admissible (e.g. $(\cdot$, inc $, 1, \cdot)$ represents any instruction incrementing the first counter). Finally $\sigma$ denotes an arbitrary letter in the alphabeth of the player.

\footnotetext{
${ }^{4}$ The entering node (owned by Player $I$, with an incoming edge labeled start) is supposed to be connected to $q_{I}$ (the initial state of $A$, owned by Player $I$ ) by means of a transition on the symbol go $\in \Sigma_{I}$ with weight 0
} 


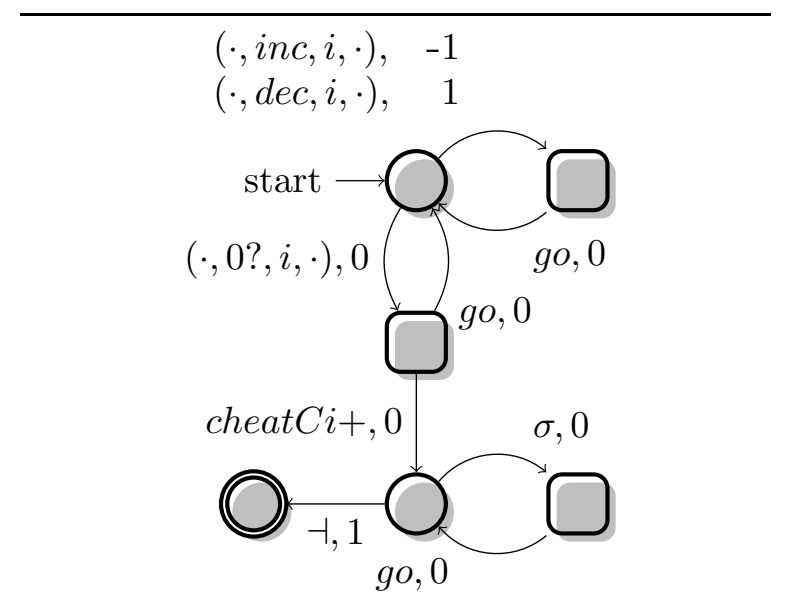

Figure 2: Gadget to check positive cheats $(\cdot, 0 ?, i, \cdot), \quad 0$ $(\cdot$, inc $, i, \cdot), \quad 1$

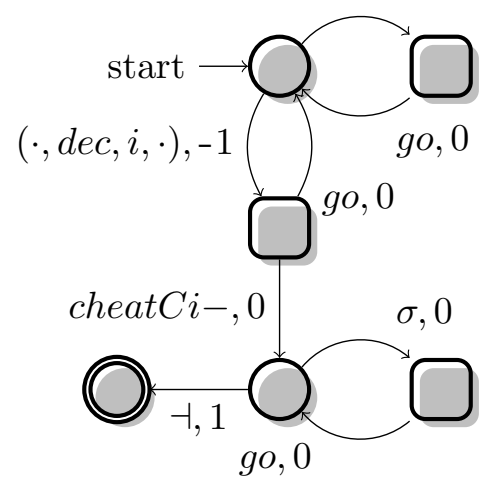

Figure 3: Gadget to check negative cheats

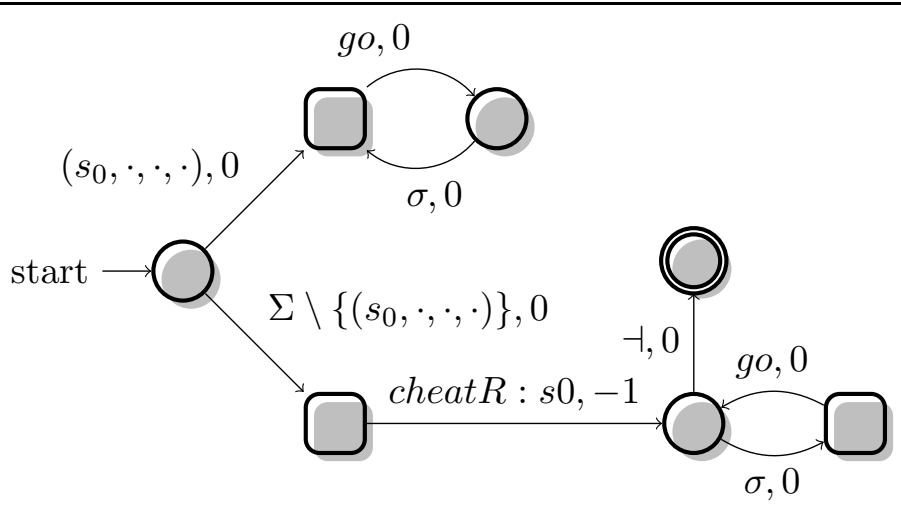

Figure 4: Gadget to check that Player $O$ plays $\left(s_{0}, \cdot, \cdot, \cdot\right)$ at the beginning. 


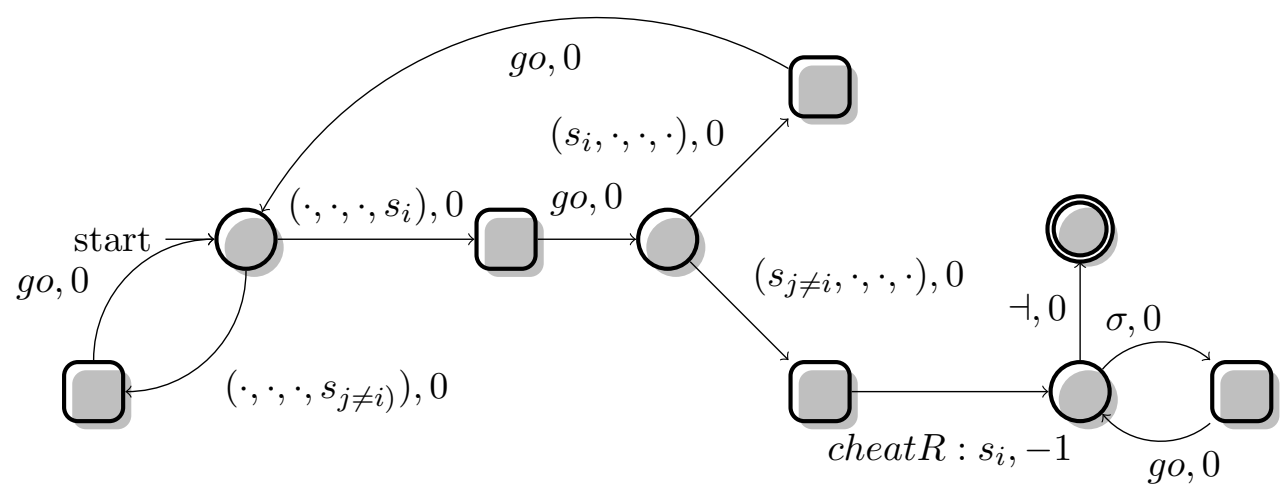

Figure 5: Gadget to check cheats along the run.

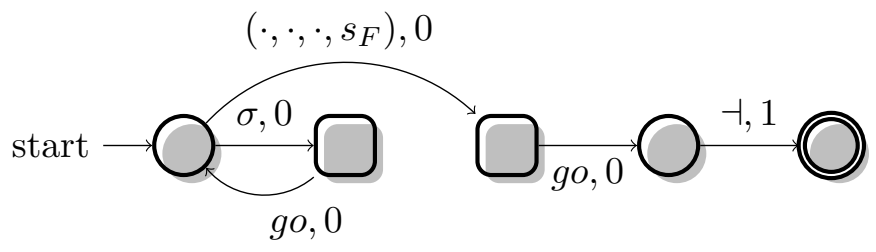

Figure 6: Neutral gadget.

Given the above notation, Figure 2 represents the gadget to check a positive cheat on counter $i, i \in\{1,2\}$. Player $I$ observes the inverted value of the counter $i$ throughout the path on $M$ simulated by Player $O$. Whenever Player $O$ declares that counter $i$ is equal to 0, Player $I$ can use the action cheatCi+ to kill all the runs in $A$ but the one within the observing gadget. The evolution of such a run up to cheatCi+ will have a negative value (corresponding to the inverted value of the observed counter) if Player $O$ was cheating. Hence, as soon as Player $O$ playes $\dashv$ it will end in a final state with weight $\leq 0$. Symmetrically, the gadget for checking negative cheats (represented in Figure 3) uses the weights on the edges to store the value of the observed counter. If Player $O$ cheats decrementing counter $i$ when its value is 0 , Player $I$ can use the action cheat $C i$ - to kill all the runs but the one (with negative value) in the gadget observing negative cheats.

Player $I$ can use the gadgets in Figures 45 to detect any structural cheat committed by Player $O$. If Player $O$ initially provides an action different from $\left(s_{0},,_{-},{ }_{-}\right)$, Player $I$ can punish him by playing action cheatR:s0. Similarly, if Player $O$ provides two actions that do not induce a (sub)-path in $M$, Player $I$ can punish him within the gadget in Figure 5. Finally, Figure 6 illustrates the neutral gadget, where Player $I$ simply observes the run provided by Player $O$ and let such a run to reach a final state as soon as Player $O$ provides an action simulating a step toward the halting state of $M$.

Theorem 5.1. Let $V \in\{$ Sum, Avg, Ratio\}. The realizability problem for functional $V$ automata is undecidable. 
Proof. We show that the encoding outlined above is correct by proving that $M$ halts iff Player $O$ has a strategy to win the realizability game on the Sum-automaton (resp. Avgautomaton) $A$. Namely, we show that Player $O$ wins the realizability game iff he provides a word $\pi$ which corresponds to an accepting run of $M$ (and then stop the game).

$(\Rightarrow)$ Suppose that $M$ halts. Let $\pi$ be the run of $M$ leading to the halting state, and consider $\lambda_{O}(\pi) \in \Lambda_{O}$, where $\lambda_{O}(\pi)$ denotes the strategy for Player $O$ induced by $\pi$, in which Player $O$ provides the word $\pi$ and then stop the game. Let $\lambda_{I} \in \Lambda_{I}$. There are two cases to consider.

(1) In the first case, $\lambda_{I}$ does not provide any action in:

$$
\left(\bigcup_{i=1,2}\{\text { cheatCi+, cheatC } i-\}\right) \cup\left(\bigcup_{0 \leq j<|S|}\left\{\text { cheatR: } s_{j}\right\}\right)
$$

Then, the only run to a final state in $A$ is the one within the neutral gadget, having weight strictly positive.

(2) In the second case, $\gamma_{I}$ contains an action in $\left(\bigcup_{i=1,2}\{\right.$ cheatCi+, cheatCi- $\left.\}\right)$. Let $\alpha$ be the first action in $\left(\bigcup_{i=1,2}\{\right.$ cheatCi+, cheat $i$ - $\left.\}\right)$ on $\lambda_{I}$. There is only one gadget allowing a run containing $\alpha$. Since $\pi$ is faithfully simulating $M$, such a run leads to a final state in the corresponding gadget with value $>0$.

Note that $\lambda_{I}$ cannot contain an action $\alpha \in\left(\bigcup_{0 \leq j<|S|}\left\{\right.\right.$ cheatR: $\left.\left.s_{j}\right\}\right)$. In fact, Player $I$ can never play cheatR: $s_{j}$, since Player $O$ does not commit any structural cheat on the run $\pi$. Hence, we conclude that $\forall \lambda_{I} \in \Lambda_{I}\left(L_{A}\right.$ (outcome $\left.\left.\left(\lambda_{O}(\pi), \lambda_{I}\right)>0\right)\right)$.

$(\Leftarrow)$ Suppose that $\lambda_{O} \in \Lambda_{O}$ is such that $\forall \lambda_{I} \in \Lambda_{I}\left(L_{A}\right.$ (outcome $\left.\left.\left(\lambda_{O}, \lambda_{I}\right) \geq 0\right)\right)$. By construction of $A, \lambda_{O}$ allows Player $O$ to survive in the gadgets for detecting positive, negative or structural cheats if and only if the projection of the outcome onto $\Sigma_{O}$ is a faithful simulation of a run in $M$. If Player $I$ can not use an action in $\left(\bigcup_{i=1,2}\{\right.$ cheatCit, cheatCi- $\left.\}\right) \cup$ $\left(\bigcup_{0 \leq j<|S|}\left\{\right.\right.$ cheatR: $\left.\left.s_{j}\right\}\right)$ to win (using the gadget targeted to check the corresponding cheat), the only remaining strategy for Player $I$ is playing indefinitely $\neg$ cheat. In that case, Player $O$ wins only if he eventually provides an action simulating a step leading to an halting state in $M$ (and then stop the game). Thus, our hypothesis entail that $\lambda_{O}$ consists in providing a run for $M$ that leads to a final state, witnessing that $M$ halts. Undecidability for Ratioautomata follows from the fact that any Avg-automaton $A=\left(Q, q_{I}, F, \delta, \gamma\right)$ can be coded into a Ratio-automaton by reweighting from $\gamma(t)$ to $(\gamma(t), 1)$ each transition $t \in \delta$.

5.2. Realizability on Deterministic Weighted Automata. The encoding in the previous subsection relies on the use of a nondeterminism. When the automata are deterministic, we recover decidability by considering suitable variants of classical games played on graphs, and prove that they are solvable in NP $\cap$ coNP.

Theorem 5.2. Let $V \in\{$ Sum, Avg, Dsum, Ratio $\}$. The realizability problem for deterministic $V$-automata is in NP $\cap$ coNP.

Proof. We first consider the case of deterministic Sum-automata. Let $A=\left(Q=Q_{O} \uplus\right.$ $\left.Q_{I}, q_{I}, F, \delta, \gamma\right)$ be a deterministic Sum-automaton. Without loss of generality, we assume that $A$ contains only one accepting state denoted by $f$ which is absorbing 5 . Then we consider $A$ as a finite state game arena and compute the set of states $S \subseteq Q$ from which player $O$

\footnotetext{
${ }^{5}$ Note that in our setting Player $\mathrm{O}$ has the ability to stop the game by means of the special symbol $\dashv$. Therefore, it is sufficient to redirect the transition labeled by $\dashv$ to a unique absorbing final state.
} 
can force a visit to the accepting state $f$. Note that from any state $s$ in $S$, player $O$ has a strategy to force a visit to $f$ within $n$ steps, where $n=|Q|$. Note also that by determinacy, the complement of this set is the set of states of $A$ from which player $I$ has a strategy to prevent a visit to $f$. Clearly, player $O$ has to avoid the states in $Q \backslash S$ at all cost and so they can be removed from $A$. Let $A^{\prime}$ be $A$ where we have kept only the states in $S$.

Now, we construct from $A^{\prime}$ a finite tree as follows. We unfold $A^{\prime}$ and stop a branch at a node when:

- it is labeled with $f$ and the sum of the weights on the branch up to the node is equal to $c>\nu$,

- it is labeled by a state $q$ that already appears on the branch from the root to the node. We call the node where $q$ already appears the ancestor of the leaf.

Let us note $L$ the set of leafs of this finite tree. We then partition the leafs of this tree into $L_{1}$, the set of leafs that are good for player $O$ and $L_{2}$, the set of leafs that are good for player $I . L_{1}$ contains:

- $\left(C_{1}\right)$ the leafs that are annotated with $f$ and for which the sum of weights is strictly greater than the realizability threshold $\nu$, and

- $\left(C_{2}\right)$ the leafs labeled with a repeating state and for which the sum of weights from the root to the leaf is strictly larger than the sum of weights from the root to the ancestor.

$L_{2}=Q \backslash L_{1}$ are the leafs that are good for player $I$. Now, consider the game played on this finite tree where player $O$ wants to reach $L_{1}$ and player $I$ wants to reach $L_{2}$. The winner in this game can be found by backward induction. We claim (and prove) below that player $I$ wins in this finite game tree iff he wins the original game.

Assume that player $O$ wins the finite game tree. We show how to construct a winning strategy in the original game. The strategy is built as follows. In the original game, player $O$ plays as in the final tree up to the point where he reaches a leaf (in $L_{1}$ ). If the leaf is of sort defined in $C_{1}$ above then we know that player $O$ has won the original game. Otherwise, we know that the sum now is strictly greater than the sum up to the ancestor of the leaf that we have reached. Then player $O$ continues to play as prescribed by its winning strategy in the tree from the ancestor. Continuing like that, each time that the game arrives at a leaf, the sum of weights has strictly increased from the last visit to that leaf. As a consequence, after a finite amount of time, the sum will be strictly larger than $\nu+n \cdot|-W|$ where $-W$ is the smallest negative weight in $A^{\prime}$. From that point, player $O$ can use his strategy that forces the state $f$ and reach it with a sum that is strictly greater than $\nu$ (this is because he can force $f$ within $n$ steps).

Now assume that player $I$ wins the finite game tree. We show how to construct a winning strategy in the original game. The strategy simply follows the strategy of player $I$ in the finite tree by applying the strategy from the ancestor when reaching a leaf. As only leaf in $L_{2}$ are reached when playing that way, we know that the sum on successive visits to repeating states is non-increasing. As a consequence, as player $O$ can not force a visit to a node labeled with $f$ and sum greater than $\nu$ in the finite game tree, we know that this will not happen in the original game neither when player $I$ plays its strategy.

This proof establishes that the realizability problem is decidable for deterministic Sumautomata. Note that player $O$ needs memory to win in the original game as he has to verify that he has reached a sufficiently high sum before applying the strategy that forces the visit to $f$. To provide the complexity bound of NP $\cap$ coNP, we reduce the realizability problem for deterministic Sum-automata to the decision problem on mean-payoff games [18, 39]. A 
mean-payoff game is a zero-sum game involving two players (the maximazer $P_{0}$ and the minimizer $P_{1}$ ) where $P_{0}$ has the following objective: to maximize his mean-payoff, defined as the liminf of the ratio between the cost of a play-prefix of length $l$ (i.e. the sum of the weights along the path) and $l$ when $l \rightarrow+\infty$.

Given a deterministic Sum-automaton $A=\left(Q=Q_{O} \uplus Q_{I}, q_{I}, F, \delta, \gamma\right)$, where $f$ is an absorbing accepting state, consider the mean-payoff game $G=\left(V_{0}, V_{1}, \delta_{G}, \gamma\right)$, where $V_{0} \subseteq Q_{O}$ (resp. $\left.V_{1} \subseteq Q_{I}\right)$ is the set of states of player $O$ (resp. player $I$ ) for which player $O$ has a strategy to force a visit to $f$. The transition relation $\delta_{G}$ is built as follows. We add an edge from $f$ back to $q_{I}$ (weighted 0 ) and we omit the self-loop on the absorbing final state. Moreover, if $\nu>0$ then $\delta_{G}\left(q, q^{\prime}\right)=\delta\left(q, q^{\prime}\right)-\nu$ for each edge $\left(q, q^{\prime}\right)$ in $A$. We prove that player $O$ has a winning strategy w.r.t. realizability on $A$ if and only if $\operatorname{val}\left(q_{I}\right)>0$ in the mean-payoff game $G$. Suppose $\operatorname{val}\left(q_{I}\right)>0$ in $G$. Then, player $O$ has a memoryless strategy to ensure $\operatorname{val}\left(q_{I}\right)>0$. Let $\sigma$ be such a memoryless strategy for player $O$, and consider the graph $G_{\sigma}$ obtained from $G$ by removing all the edges sourcing from a vertex in $V_{0}$ that are not chosen according to $\sigma$. Since $\sigma$ guarantees $\operatorname{val}\left(q_{I}\right)>0, G_{\sigma}$ does not contain any non-positive cycle. By construction, each simple cycle involving $f$ in $G$ is a simple path to $f$ followed by an edge back to $q_{I}$ with weight 0 . Hence, $G_{\sigma}$ contains a cycle through $f$ if and only if $G$ contains a simple positive path to $f$. Therefore, playing according to $\sigma$ on $A$, player $O$ will either reach $f$ with sum greater than $\nu$, or he will eventually reach a sum high enough to guarantee him to win, as soon as he forces the play to reach $f$. Conversely, assume that player $O$ has a strategy to win the realizability game on $A$ and let $\sigma$ be a corresponding winning strategy. To win the mean-payoff game $G$, player $O$ needs simply to apply $\sigma$ on $G$, since this leads him to $f$ with a positive sum, and then the play starts back from $q_{I}$ following the only edge sourcing from $f$ back to $q_{I}$. As shown in the previous results Avg games can be reduced easily to Sum games, and as for the questions about thresholds, Ratio games can be reduced to Avg games.

We now turn to the case of Dsum. The solution for Dsum is obtained by first removing from $A$ all states from which player $O$ cannot force a visit to $f$. As above, we note the game where those states have been removed by $A^{\prime}$. Then, we consider $A^{\prime}$ as an (infinite) discounted sum game where player $O$ tries to maximize the value of the discounted sum while player $I$ tries to minimize this value. Let $v$ denote the value of the initial state $q_{I}$ in that game. We claim that player $O$ wins the initial game iff the value $v$ in $q_{I}$ is such that $v>\nu$. Indeed, if player $O$ has a winning strategy in the original game, i.e. a strategy to force the game into $f$ with discounted sum strictly greater than $\nu$, then by playing this strategy in the discounted sum game, the infinite discounted sum will be equal to the discounted sum up to $f$ as from there only the self loop on $f$ is traversed and its weight is equal to 0 . Now assume that player $O$ has a strategy that force a value $v>\nu$ in the discounted sum game. Then by playing that strategy for $i$ steps in the original game with $i$ large enough to make sure that $\lambda^{i} W+\cdots+\lambda^{i+n} W$ is small enough, he will be able to switch to its strategy that forces $f$ after at most $n$ steps and ensure to reach $f$ with a discounted sum $>\nu$. As infinite discounted sum games are in NP $\cap$ coNP [3] and since our reduction is polynomial, we also get that finite reachability discounted sum games are in NP $\cap$ coNP. 


\section{Determinizability Problem}

A weighted $V$-automaton $A$ is said to be determinizable it there exists a deterministic $V$ automaton $D$ such that $L_{A}(w)=L_{D}(w)$ for all $w \in \Sigma_{-}^{+}$. Weighted automata are not determinizable in general. For example, consider the right automaton on Fig. 1. Seen as Sum-automaton, it cannot be determinized, because there are infinitely many delays associated with the pair of states $(p, q)$. Those delays can for instance be obtained by the family of words of the form $a^{n}$. Determinizability is already known to be decidable in PTime for functional Sum-automata 23]6. It is also known that any Dsum-automaton (even nonfunctional) with an integral discount factor, i.e. $\lambda=1 / n$, where $n>1$ is an integer, is determinizable [10.

Determinizable functional Sum-automata are characterized by the so called twinning property, that has been introduced for finite word transducers [13, 4]. In particular, the twinning property has been used as a sufficient condition for the termination of Mohri's determinization algorithm [16] for (non-functional) weighted automata over the tropical semiring. In [23], Kirsten et al. proved that such a property is also a necessary condition for determinization of functional Sum-automata. The twinning property has been also used as a sufficient condition for the termination of a determinization procedure, applied to classes of weighted automata defined on more general commutative semirings [23] (the commutativity hypothesis is necessary here to ensure that the twinning property is sufficient). In this paper, we consider determinization of functional arbitrary group automata. Further, we show that the twinning property is also a necessary condition for the determinization of functional group automata over groups enjoying the so called infinitary property. We show that the groups encoding Sum, Avg and Dsum are all infinitary. Therefore, our general decidability result for the determinization problem on functional infinitary group automata applies to functional Sum, Avg and Dsum-automata.

6.1. Determinization of functional group automata. We first define a determinization construction for functional group automata. This construction does not necessarily yield a deterministic group automaton with a finite set of states, unless, as we show in Subsection 6.2, the twinning property is satisfied.

The procedure is similar to the one of [16] for weighted automata over a semiring (under some conditions, such as weak divisibility), and to that of finite-state transducers [13, 4], but adapted to groups. In particular, the procedure of [16] heavily uses the additive operation of semi-rings, and the determinization of finite-state transducers relies on the operation of taking the longest common prefix of two strings. One cannot rely on similar operations for group automata. To be more precise, both known determinization procedures in [13, 4, 16] extend the classical subset construction with delays (what remains to be output). States are therefore pairs $(q, d)$ where $q$ is a state of the original automaton, and $d$ is a delay (unlike functional automata, there can be several delays associated with the same state). Initially, all delays are equal to $\mathbb{1}$. When reading a new symbol $a$ from a state $P$, the deterministic automaton outputs a value $v$ and goes to a state $P^{\prime}$. Let us explain how $v$ is computed. Let $T$ be the set of transitions $\left(p, a, p^{\prime}\right)$ of the original automaton on $a$, such that there exists $(p, d) \in P$. Let $\gamma\left(p, a, p^{\prime}\right)$ be their associated value. The value $v$ is obtained by taking the sum (resp. the longest common prefix) of the set $\left\{d \cdot \gamma\left(p, a, p^{\prime}\right) \mid(p, d) \in P\right\}$. In other words, for each transition $\left(p, a, p^{\prime}\right)$, what remains to be output is $d \cdot \gamma\left(p, a, p^{\prime}\right)$, and the output of

\footnotetext{
${ }^{6}$ See 24, 22] for determinizability results on more general classes of Sum-automata.
} 
the deterministic automaton is somehow the "best" that can be output at the moment: the sum (or longest common prefix respectively) of all these values. The state $P^{\prime}$ is then the set of states $p^{\prime}$ with updated delays, i.e. what still remain to be output.

Our determinization construction differs from the procedures in [13, 4, 16] sketched above in two points: $(i)$ it applies to group automata that are functional, and therefore there is at most one delay per state (otherwise, since we assume that all state are co-accessible, functionality would be falsified) ( $i i$ ) to compute the value $v$, since we cannot rely on the additive operation, nor the longest common prefix operation, we pick a base transition $\left(p_{0}, a, p_{0}^{\prime}\right)$ (according to a given total order on transitions), and $v$ is then $d \cdot \gamma\left(p_{0}, a, p_{0}^{\prime}\right)$, where $d$ is the delay associated with $p_{0}$. The new delays are computed relatively to $v$. The construction works for any order on transitions, possibly yielding different deterministic automata?.

Given the above premises, let us describe our determinization construction formally. Let $A=\left(Q, q_{I}, F, \delta, \gamma\right)$ be a trim functional group automaton over a group $(W, \cdot, \mathbb{1})$ and assume, w.l.o.g., that $\delta$ is totally ordered by some order $\leq_{\delta}$. The output of our construction is a group automaton $A_{d}=\left(Q_{d}, f_{I}, F_{d}, \delta_{d}, \gamma_{d}\right)$ over $(W, \cdot, \mathbb{1})$ such that $L_{A_{d}}=L_{A}$ and $\delta_{d}$ is deterministic. However, $A_{d}$ is not a proper deterministic group automaton, as it may have infinitely many states. The next section gives a sufficient condition under which $Q_{d}$ needs to be finite. Let $\mathcal{D}$ be the set of delays delay $\left(\rho, \rho^{\prime}\right)$ for any two runs $\rho, \rho^{\prime}$ of $A$ on the same word. We define $Q_{d}=\mathcal{D}^{Q}$, as the set of partial functions from states $Q$ to delays. We let $f_{I}: q_{I} \mapsto \mathbb{1}$ be the initial function, defined for $q_{I}$ only. The set of accepting states $F_{d}$ is defined as $\left\{f \in Q_{d} \mid \operatorname{dom}(f) \cap F \neq \varnothing\right\}$. Then, given partial functions $f, f^{\prime} \in Q_{d}$ and a symbol $a \in \Sigma$, we let $t_{0}=\left(p_{0}^{\prime}, a, p_{0}\right)$ be the smallest transition (for $\leq_{\delta}$ ) from a state $p^{\prime} \in \operatorname{dom}\left(f^{\prime}\right)$ to a state $p_{0} \in \operatorname{dom}(f)$ on $a$. Let $v_{0}={ }_{\operatorname{def}} f^{\prime}\left(p^{\prime}\right) \cdot \gamma\left(t_{0}\right)$. Then, we let $\left(f^{\prime}, a, f\right) \in \delta_{d}$ if for all transitions $\left(q^{\prime}, a, q\right) \in \delta$ such that $q^{\prime} \in \operatorname{dom}\left(f^{\prime}\right), f(q)$ is defined and equals

$$
f(q)=v_{0}^{-1} \cdot f^{\prime}\left(q^{\prime}\right) \cdot \gamma\left(q^{\prime}, a, q\right)
$$

The value $\gamma_{d}\left(f^{\prime}, a, f\right)$ of the transition $\left(f^{\prime}, a, f\right)$ is $v_{0}$. Note that $A_{d}$ is deterministic, because $f$ is functionally obtained from $f^{\prime}$ and $a$.

Lemma 6.1. Let $A$ be a trim functional group automaton and $A_{d}$ be the group automaton obtained by determinization. Then, for all $w \in \Sigma^{+}, L_{A}(w)=L_{A_{d}}(w)$. However, $A_{d}$ may have infinitely many states.

Proof. We show that $A_{d}$ maintains the following invariant: for all $w \in \Sigma^{*}$ such that there is a run $\rho_{d}: f_{I} \stackrel{w}{\rightarrow} f \in Q_{d}$, the following three properties hold:

(1) there exists $p_{0} \in \operatorname{dom}(f)$ such that $f\left(p_{0}\right)=\mathbb{1}$,

(2) for all $q \in \operatorname{dom}(f)$, for all runs $\rho: q_{I} \stackrel{w}{\rightarrow} q$ and $\rho_{0}: q_{I} \stackrel{w}{\rightarrow} p_{0}, f(q)=\operatorname{delay}\left(\rho_{0}, \rho\right)$,

(3) for all $q \in \operatorname{dom}(f)$, for all runs $\rho: q_{I} \stackrel{w}{\rightarrow} q, V(\rho)=V\left(\rho_{d}\right) \cdot f(q)$.

We show this invariant by induction on $|w|$. It is clearly true when $|w|=0$. Suppose that $w=w^{\prime} a$, where $a \in \Sigma$, and let $f^{\prime} \in Q_{d}$ be such that $f_{I} \stackrel{w}{\rightarrow} f^{\prime}$ and $\left(f^{\prime}, a, f\right) \in Q_{d}$. We show the two conditions:

(1) it suffices to take $p_{0}$ as defined in the determinization construction, because by definition $f\left(p_{0}\right)=v_{0}^{-1} \cdot v_{0}=\mathbb{1}$.

\footnotetext{
${ }^{7}$ While point $(i)$ could be relaxed by having sets of delays associated with each state, and several outputs on the terminal symbol $\dashv$, we rather assume functionality as it is the purpose of this paper, and it lightens our notations.
} 
(2) Let $\rho^{\prime}: q_{I} \stackrel{w}{\rightarrow} q^{\prime}$ be a run such that $\rho^{\prime} \cdot\left(q^{\prime}, a, q\right)=\rho$, and let $\rho_{0}^{\prime}: q_{I} \stackrel{w}{\rightarrow} p^{\prime}$ be a run, where $p^{\prime}$ is defined as in the definition of $\delta_{d}$. By induction hypothesis, by (1), there exists $p_{0}^{\prime} \in Q$ such that $f^{\prime}\left(p_{0}^{\prime}\right)=\mathbb{1}$. Let $r: q_{I} \stackrel{w}{\rightarrow} p_{0}^{\prime}$ be a run of $A$ on $w$. Again by induction hypothesis, by $(3)$, we have $f^{\prime}\left(p^{\prime}\right)=\operatorname{delay}\left(r, \rho_{0}^{\prime}\right)$ and $f^{\prime}\left(q^{\prime}\right)=\operatorname{delay}\left(r, \rho^{\prime}\right)$, i.e. $f^{\prime}\left(p^{\prime}\right)=V(r)^{-1} \cdot V\left(\rho_{0}^{\prime}\right)$ and $f^{\prime}\left(q^{\prime}\right)=V(r)^{-1} \cdot V\left(\rho^{\prime}\right)$. Now, by definition of $\delta_{d}$, we have:

$$
\begin{aligned}
f(q) & =v_{0}^{-1} \cdot f^{\prime}\left(q^{\prime}\right) \cdot \gamma\left(q^{\prime}, a, q\right) \\
& =\left(f^{\prime}\left(p^{\prime}\right) \cdot \gamma\left(p^{\prime}, a, p_{0}\right)\right)^{-1} \cdot f^{\prime}\left(q^{\prime}\right) \cdot \gamma\left(q^{\prime}, a, q\right) \\
& =\gamma\left(p^{\prime}, a, p_{0}\right)^{-1} \cdot\left(f^{\prime}\left(p^{\prime}\right)\right)^{-1} \cdot f^{\prime}\left(q^{\prime}\right) \cdot \gamma\left(q^{\prime}, a, q\right) \\
& =\gamma\left(p^{\prime}, a, p_{0}\right)^{-1} \cdot\left(V(r)^{-1} \cdot V\left(\rho_{0}^{\prime}\right)\right)^{-1} \cdot V(r)^{-1} \cdot V\left(\rho^{\prime}\right) \cdot \gamma\left(q^{\prime}, a, q\right) \\
& =\gamma\left(p^{\prime}, a, p_{0}\right)^{-1} \cdot V\left(\rho_{0}^{\prime}\right)^{-1} \cdot V(r) \cdot V(r)^{-1} \cdot V\left(\rho^{\prime}\right) \cdot \gamma\left(q^{\prime}, a, q\right) \\
& =\gamma\left(p^{\prime}, a, p_{0}\right)^{-1} \cdot V\left(\rho_{0}^{\prime}\right)^{-1} \cdot V\left(\rho^{\prime}\right) \cdot \gamma\left(q^{\prime}, a, q\right) \\
& =V\left(\rho_{0}\right)^{-1} \cdot V(\rho) \\
& =\operatorname{delay}\left(\rho_{0}, \rho\right)
\end{aligned}
$$

(3) Let $\rho_{d}^{\prime}$ be the run of $A_{d}$ on $w$. Let $\rho^{\prime}: q_{I} \stackrel{w}{\rightarrow} q^{\prime}$ be such that $\rho^{\prime} \cdot\left(q^{\prime}, a, q\right)=\rho$. By induction hypothesis and (3), we know that

$$
V\left(\rho^{\prime}\right)=V\left(\rho_{d}^{\prime}\right) \cdot f^{\prime}\left(q^{\prime}\right)
$$

and therefore since $V\left(\rho_{d}\right)=V\left(\rho_{d}^{\prime}\right) \cdot v_{0}$,

$$
V\left(\rho^{\prime}\right)=V\left(\rho_{d}\right) \cdot v_{0}^{-1} \cdot f^{\prime}\left(q^{\prime}\right)
$$

From which we get

$$
V\left(\rho^{\prime}\right) \cdot \gamma\left(q^{\prime}, a, q\right)=V\left(\rho_{d}\right) \cdot v_{0}^{-1} \cdot f^{\prime}\left(q^{\prime}\right) \cdot \gamma\left(q^{\prime}, a, q\right)
$$

which, since $V(\rho)=V\left(\rho^{\prime}\right) \cdot \gamma\left(q^{\prime}, a, q\right)$ and $f(q)=v_{0}^{-1} \cdot f^{\prime}\left(q^{\prime}\right) \cdot \gamma\left(q^{\prime}, a, q\right)$, is equivalent to $V(\rho)=V\left(\rho_{d}\right) \cdot f(q)$.

From this invariant, it is not difficult to show that $L_{A}=L_{A_{d}}$. Indeed, let $w \in \operatorname{dom}(A)$, let $\rho$ be an accepting run of $A$ on $w$ (therefore $V(\rho)=L_{A}(w)$ ), and let $\rho_{d}$ be the run of $A_{d}$ on $w$ (its existence is already a consequence of the subset construction in finite automata). Clearly, $\rho_{d}$ ends in a state $f$ such that $\operatorname{dom}(f) \subseteq F$, because $w$ necessarily ends with $\dashv$, and it is the only way in $A$ to reach an accepting state. By invariant (2), $f(q)=\operatorname{delay}\left(\rho_{0}, \rho\right)$ for $\rho_{0}$ a run of $A$ on $w$ ending in a state $p_{0}$ such that $f\left(p_{0}\right)=\mathbb{1}$. By invariant (3), $V\left(\rho_{d}\right)=V\left(\rho_{0}\right) \cdot f\left(p_{0}\right)=V\left(\rho_{0}\right)$. Since $\operatorname{dom}(f) \subseteq F, \rho_{0}$ is accepting, and since $A$ is functional, $V\left(\rho_{0}\right)=V(\rho)$. Therefore $V(\rho)=V\left(\rho_{d}\right)$, i.e. $L_{A_{d}}(w)=L_{A}(w)$. The converse is shown similarly.

Note that for finite groups, this determinization always yields an equivalent (finite) deterministic group automaton, because the set of delays is finite.

Lemma 6.2. Any functional group automaton over a finite group is determinizable.

6.2. The twinning property: a sufficient condition for determinizability. Lemma 6.4 shows that the twinning property (cfr. Definition 6.3) is sufficient to guarantee the termination of the determinization construction, i.e., the finiteness of the automaton $A_{d}$ obtained by determinization. 
Definition 6.3. Two states $p, q$ of a functional group automaton $A$ are twinned if both $p$ and $q$ are co-accessible and for all words $w_{1}, w_{2} \in \Sigma^{*}$, for all runs $\rho_{1}: q_{I} \stackrel{w_{1}}{\longrightarrow} p, \rho_{2}: p \stackrel{w_{2}}{\longrightarrow} p$, $\rho_{1}^{\prime}: q_{I} \stackrel{w_{1}}{\longrightarrow} q, \rho_{2}^{\prime}: q \stackrel{w_{2}}{\longrightarrow} q$, we have $\operatorname{delay}\left(\rho_{1}, \rho_{1}^{\prime}\right)=\operatorname{delay}\left(\rho_{1} \rho_{2}, \rho_{1}^{\prime} \rho_{2}^{\prime}\right)$. The automaton $A$ satisfies the twinning property if all pairs of states are twinned.

Lemma 6.4. Let $A$ be a group automaton. If $A$ satisfies the twinning property, then there are at most $|\Sigma|^{|Q|^{2}}$ delays delay $\left(\rho, \rho^{\prime}\right)$ for any two runs $\rho, \rho^{\prime}$ on the same input, and thus the deterministic group automaton $A_{d}$ obtained by the determinization procedure is finite.

Proof. As delays must be equivalent on parallel loops, any delay can be obtained with some pair of runs of length $|Q|^{2}$ at most (on longer pairs of runs, there must exist a parallel loop with equivalent delays that can be removed without affecting the value of the global delay of both runs, see Lemma 3.3. .

The following lemma states a short witness property for the non satisfiability of the twinning property. This result is crucial to prove that the twinning property is decidable (cfr. Lemma 6.6).

Lemma 6.5. If a functional group automaton $A$ does not satisfy the twinning property, there exist two words $w_{1}, w_{2} \in \Sigma^{*}$ such that $\left|w_{1}\right| \leq 2|Q|^{2}$ and $\left|w_{2}\right| \leq 2|Q|^{2}$, two states $p, q \in Q$ such that $p$ and $q$ are both co-accessible, and runs $\rho_{1}: q_{I} \stackrel{w_{1}}{\longrightarrow} p, \rho_{2}: p \stackrel{w_{2}}{\longrightarrow} p$, $\rho_{1}^{\prime}: q_{I} \stackrel{w_{1}}{\longrightarrow} q, \rho_{2}^{\prime}: q \stackrel{w_{2}}{\longrightarrow} q$, such that delay $\left(\rho_{1}, \rho_{1}^{\prime}\right) \neq \operatorname{delay}\left(\rho_{1} \rho_{2}, \rho_{1}^{\prime} \rho_{2}^{\prime}\right)$.

Proof. Suppose that $\left|w_{2}\right|>2|Q|^{2}$ (the case $\left|w_{1}\right|>2|Q|^{2}$ is proved exactly the same way) and that $w_{1} w_{2}$ witnesses that the twinning property does not hold by the decomposition into runs $\rho_{1}, \rho_{2}, \rho_{1}^{\prime}, \rho_{2}^{\prime}$ as in the premisses of the lemma. We will show that we can shorten the runs $\rho_{1}, \rho_{1}^{\prime}$ and still get a witness that the twinning property does not hold.

Since $\left|w_{2}\right|>2|Q|^{2}$, there is a pair of states $\left(p^{\prime}, q^{\prime}\right)$ that repeats three times along the two parallel runs $\rho_{2}$ and $\rho_{2}^{\prime}$, i.e. $w_{2}$ can be decomposed as $w_{1}^{\prime} w_{2}^{\prime} w_{3}^{\prime} w_{4}^{\prime}$ and $\rho_{2}$ and $\rho_{2}^{\prime}$ can be decomposed as $r_{1} r_{2} r_{3} r_{4}$ and $r_{1}^{\prime} r_{2}^{\prime} r_{3}^{\prime} r_{4}^{\prime}$ respectively, where:

$$
\begin{array}{llll}
r_{1}: p \stackrel{w_{1}^{\prime}}{\longrightarrow} p^{\prime} & r_{2}: p^{\prime} \stackrel{w_{2}^{\prime}}{\longrightarrow} p^{\prime} & r_{3}: p^{\prime} \stackrel{w_{3}^{\prime}}{\longrightarrow} p^{\prime} & r_{4}: p^{\prime} \stackrel{w_{4}^{\prime}}{\longrightarrow} p \\
r_{1}^{\prime}: q \stackrel{w_{1}^{\prime}}{\longrightarrow} q^{\prime} & r_{2}^{\prime}: q^{\prime} \stackrel{w_{2}^{\prime}}{\longrightarrow} q^{\prime} & r_{3}^{\prime}: q^{\prime} \stackrel{w_{3}^{\prime}}{\longrightarrow} q^{\prime} & r_{4}^{\prime}: p^{\prime} \stackrel{w_{4}^{\prime}}{\longrightarrow} q
\end{array}
$$

Note that $r_{1}, r_{1}^{\prime}$ and $r_{4}, r_{4}^{\prime}$ may be empty (in this case $p=p^{\prime}$ and $q=q^{\prime}$ ), but $r_{2}, r_{3}, r_{2}^{\prime}, r_{3}^{\prime}$ are assumed to be non-empty.

Now, there are two cases: delay $\left(\rho_{1} r_{1}, \rho_{1}^{\prime} r_{1}^{\prime}\right) \neq \operatorname{delay}\left(\rho_{1} r_{1} r_{2}, \rho_{1}^{\prime} r_{1}^{\prime} r_{2}^{\prime}\right)$ and in that case the word $w_{1} w_{1}^{\prime} w_{2}^{\prime}$ is a witness that the twinning property does not hold, and $\left|w_{1} w_{1}^{\prime} w_{2}^{\prime}\right|<\left|w_{1} w_{2}\right|$. In the second case, we have delay $\left(\rho_{1} r_{1}, \rho_{1}^{\prime} r_{1}^{\prime}\right)=\operatorname{delay}\left(\rho_{1} r_{1} r_{2}, \rho_{1}^{\prime} r_{1}^{\prime} r_{2}^{\prime}\right)$, but in that case, we can apply Lemma 3.3 and we get delay $\left(\rho_{1} r_{1} r_{3} r_{4}, \rho_{1}^{\prime} r_{1}^{\prime} r_{3}^{\prime} r_{4}^{\prime}\right)=\operatorname{delay}\left(\rho_{1} \rho_{2}, \rho_{1}^{\prime} \rho_{2}^{\prime}\right)$.

Therefore, delay $\left(\rho_{1}, \rho_{1}^{\prime}\right) \neq \operatorname{delay}\left(\rho_{1} r_{1} r_{3} r_{4}, \rho_{1}^{\prime} r_{1}^{\prime} r_{3}^{\prime} r_{3}^{\prime}\right)$ and $w_{1} w_{1}^{\prime} w_{3}^{\prime} w_{4}^{\prime}$ is a shorter witness that the twinning property does not hold.

Lemma 6.6. It is decidable in CoNP whether a functional group automaton satisfies the twinning property.

Proof. First, the automaton is transformed into a trim automaton. Then, it suffices to guess two runs on the same input word of size at most $4|Q|^{2}$ and two positions in those runs, and check (in ptime) that the pair of states at the two positions are equal and that the respective delays are different. This algorithm is correct thanks to Lemma 6.5. 
6.3. Determinization of functional infinitary group automata. In this subsection, we define the class of infinitary groups, and show that the twinning property is a necessary condition for determinizability of functional group automata over an infinitary group. Morevoer, we show that the groups encoding Sum, Avg, and Dsum (cf. Lemma 2.4) belong to this class, yielding a procedure to decide determinizability for the classes of weighted $V$-automata, for $V \in\{$ Sum, Avg, Dsum $\}$. Intuitively, the infinitary condition implies that iterating two runs of an infinitary group automaton on a parallel loop induces infinitely many delays.

Definition 6.7 (Infinitary Groups). A group $(W, \cdot, \mathbb{1})$ is said to be infinitary if it satisfies the infinitary condition stating that for all $v_{1}, w_{1}, v_{2}, w_{2} \in W$, if $v_{1}^{-1} \cdot w_{1} \neq v_{2}^{-1} \cdot v_{1}^{-1} \cdot w_{1} \cdot w_{2}$, then:

$$
\left|\left\{v_{2}^{-h} \cdot v_{1}^{-1} \cdot w_{1} \cdot w_{2}^{h} \mid h \geq 0\right\}\right|=\infty
$$

A group automaton over an infinitary group is called an infinitary group automaton.

We show that the groups encoding Sum, Avg and Dsum satisfy the infinitary condition, as well as any linearly ordered group. A linearly ordered group $(W, \cdot, \mathbb{1},<)$ is simply a group $(W, \cdot, 1)$ equipped with a strict total order $<$ such that (monotonicity) for all $x, y, l, r \in W$, if $x<y$ then $l \cdot x \cdot r<l \cdot y \cdot r$.

Proposition 6.8. Any linearly ordered group $(W, \cdot, \mathbb{1},<)$ is infinitary.

Proof. Indeed, let $v_{1}, w_{1}, v_{2}, w_{2} \in W$ such that $v_{1}^{-1} \cdot w_{1} \neq v_{2}^{-1} \cdot v_{1}^{-1} \cdot w_{1} \cdot w_{2}$. Suppose that $v_{1}^{-1} \cdot w_{1}<v_{2}^{-1} \cdot v_{1}^{-1} \cdot w_{1} \cdot w_{2}$, then by monotonicity, $v_{2}^{-1} \cdot v_{1}^{-1} \cdot w_{1} \cdot w_{2}<v_{2}^{-1} \cdot v_{2}^{-1} \cdot v_{1}^{-1} \cdot w_{1} \cdot w_{2} \cdot w_{2}$. More generally, for all $i<j, v_{2}^{-i} \cdot v_{1}^{-1} \cdot w_{1} \cdot w_{2}^{i}<v_{2}^{-j} \cdot v_{1}^{-1} \cdot w_{1} \cdot w_{2}^{j}$. The infinitary condition is shown similarly when assuming $v_{1}^{-1} \cdot w_{1}>v_{2}^{-1} \cdot v_{1}^{-1} \cdot w_{1} \cdot w_{2}$.

Since the groups encoding Sum and Avg can be linearly ordered, we immediately get that they are infinitary. For Dsum, we do a different proof.

Proposition 6.9. For $V \in\{$ Sum, Avg, Dsum $\}$, let $G_{V}$ be the groups defined in Lemma 2.4. Then $G_{V}$ is infinitary.

Proof. We show that for the three measures, the groups that encode them (see Lemma 2.4) can be linearly ordered. Then, the result follows by Proposition 6.8,

For $G_{\text {Sum }}$, it suffices to order its elements with the natural order on integers. Similarly, for $G_{\mathrm{Avg}}$, it suffices to order its elements by lexicographic order. For both orders, it is easy to show they satisfy the monotonicity property.

The group $G_{\text {Dsum }}$ can also be linearly ordered by lexicographic order as follows: $(a, x)<$ $(b, y)$ if $x<y$, or $x=y$ and $a<b$. Let us show that this order satisfies the monotonicity condition. Assume that $(a, x)<(b, y)$ and consider $(c, z) \in \mathbb{Q} \times \mathbb{Q}^{+}$. Then, $(c, z) \cdot(a, x)=$ $\left(\frac{c}{x}+a, z x\right)$ and $(c, z) \cdot(b, y)=\left(\frac{c}{y}+b, z y\right)$. Suppose that $x<y$, then clearly, since $z, x, y$ are strictly positive, we have $z x<z y$, and therefore $(c, z) \cdot(a, x)<(c, z) \cdot(b, y)$. Otherwise, if $x=y$, then $a<b$, and we get $z x=z y, \frac{c}{x}+a<\frac{c}{y}+b$, which implies again $(c, z) \cdot(a, x)<$ $(c, z) \cdot(b, y)$.

Now, we show that $(a, x) \cdot(c, z)<(b, y) \cdot(c, z)$. By definition of $\cdot(a, x) \cdot(c, z)=\left(\frac{a}{z}+c, x z\right)$ and $(b, y) \cdot(c, z)=\left(\frac{b}{z}+c, y z\right)$. If $x<y$, then clearly $x z<y z$ and we are done. Otherwise, $x=y$ and $a<b$, which implies that $\frac{a}{z}+c<\frac{b}{z}+c$. 
Lemma 6.10. Let $A$ be a trim functional infinitary group automaton. If $A$ does not satisfy the twinning property, then $A$ is not determinizable.

Proof. Suppose that the twinning property does not hold. Therefore we can find words $w_{1}, w_{2}$ and runs $\rho_{1}, \rho_{1}^{\prime}$ on $w_{1}$ and $\rho_{2}, \rho_{2}^{\prime}$ on $w_{2}$ such that $\rho_{2}, \rho_{2}^{\prime}$ are loops (on state $p$ and $q$ respectively), and delay $\left(\rho_{1}, \rho_{1}^{\prime}\right) \neq \operatorname{delay}\left(\rho_{1} \rho_{2}, \rho_{1}^{\prime} \rho_{2}^{\prime}\right)$. Let $\Delta(k)=\operatorname{delay}\left(\rho_{1}\left(\rho_{2}\right)^{k}, \rho_{1}^{\prime}\left(\rho_{2}^{\prime}\right)^{k}\right)$ for all $k \in \mathbb{N}$. By the infinitary condition, the set $\{\Delta(k) \mid k \geq 0\}$ is infinite. Let $S \subseteq \mathbb{N}$ such that for all $i, j \in S$ such that $i \neq j, \Delta(i) \neq \Delta(j)$. Note that $S$ is infinite.

Suppose that there exists a deterministic automaton $A_{d}=\left(Q_{d}, f_{I}, F_{d}, \delta_{d}, \gamma_{d}\right)$ equivalent to $A$. We exhibit a contradiction. Since $S$ is infinite and $Q_{d}$ is finite, we can find $k_{1} \neq k_{2} \in \mathbb{N}$ such that $k_{1} \in S, k_{1}+k_{2} \in S$ (i.e. $\left.\Delta\left(k_{1}\right) \neq \Delta\left(k_{1}+k_{2}\right)\right)$, and such that on $w_{1}\left(w_{2}\right)^{k_{1}+k_{2}}$, the run of $A_{d}$ has the following form:

$$
f_{I} \stackrel{w_{1}\left(w_{2}\right)^{k_{1}}}{\longrightarrow} f \stackrel{\left(w_{2}\right)^{k_{2}}}{\longrightarrow} f
$$

for some $f \in Q_{d}$.

Moreover, since $p$ and $q$ are both co-accessible, there exist two words $w_{3}, w_{3}^{\prime}$ and two runs of $A_{d}$ of the form:

$$
\begin{aligned}
& \rho_{d}: f_{I} \stackrel{w_{1}\left(w_{2}\right)^{k_{1}}}{\longrightarrow} f \stackrel{\left(w_{2}\right)^{k_{2}}}{\longrightarrow} f \stackrel{w_{3}}{\longrightarrow} g \\
& \rho_{d}^{\prime}: f_{I} \stackrel{w_{1}\left(w_{2}\right)^{k_{1}}}{\longrightarrow} f \stackrel{\left(w_{2}\right)^{k_{2}}}{\longrightarrow} f \stackrel{w_{3}^{\prime}}{\longrightarrow} g^{\prime}
\end{aligned}
$$

for some accepting states $g, g^{\prime} \in F_{d}$. Let $\rho_{d}=\rho_{d, 1} \rho_{d, 2} \rho_{d, 3}$ and $\rho_{d}^{\prime}=\rho_{d, 1} \rho_{d, 2} \rho_{d, 3}^{\prime}$ for some subruns $\rho_{d, 1}, \rho_{d, 2}$ that correspond to $w_{1}\left(w_{2}\right)^{k_{1}}$ and $\left(w_{2}\right)^{k_{2}}$ respectively, and some subruns $\rho_{d, 3}$ and $\rho_{d, 3}^{\prime}$ that correspond to $w_{3}$ and $w_{3}^{\prime}$ respectively. We know that $\Delta\left(k_{1}\right) \neq \Delta\left(k_{1}+k_{2}\right)$. We show that this leads to a contradiction. Let also $\rho_{3}: p \stackrel{w_{3}}{\longrightarrow} p_{f}$ and $\rho_{3}^{\prime}: q \stackrel{w_{3}^{\prime}}{\longrightarrow} q_{f}$ be two runs in $A$, for some $p_{f}, q_{f} \in F$. Then we have:

$$
\begin{aligned}
V\left(\rho_{d, 1} \rho_{d, 2} \rho_{d, 3}\right) & =V\left(\rho_{1}\left(\rho_{2}\right)^{k_{1}+k_{2}} \rho_{3}\right) \\
V\left(\rho_{d, 1} \rho_{d, 2} \rho_{d, 3}^{\prime}\right) & =V\left(\rho_{1}^{\prime}\left(\rho_{2}^{\prime}\right)^{k_{1}+k_{2}} \rho_{3}^{\prime}\right) \\
V\left(\rho_{d, 1} \rho_{d, 3}\right) & =V\left(\rho_{1}\left(\rho_{2}\right)^{k_{1}} \rho_{3}\right) \\
V\left(\rho_{d, 1} \rho_{d, 3}^{\prime}\right) & =V\left(\rho_{1}^{\prime}\left(\rho_{2}^{\prime}\right)^{k_{1}} \rho_{3}^{\prime}\right)
\end{aligned}
$$

From Equations 6.4 and 6.5 we get:

$$
V\left(\rho_{d, 1} \rho_{d, 2} \rho_{d, 3}^{\prime}\right)^{-1} \cdot V\left(\rho_{d, 1} \rho_{d, 2} \rho_{d, 3}\right)=V\left(\rho_{1}^{\prime}\left(\rho_{2}^{\prime}\right)^{k_{1}+k_{2}} \rho_{3}^{\prime}\right)^{-1} \cdot V\left(\rho_{1}\left(\rho_{2}\right)^{k_{1}+k_{2}} \rho_{3}\right)
$$

which is equivalent to:

$$
V\left(\rho_{d, 3}^{\prime}\right)^{-1} \cdot V\left(\rho_{d, 3}\right)=V\left(\rho_{1}^{\prime}\left(\rho_{2}^{\prime}\right)^{k_{1}+k_{2}} \rho_{3}^{\prime}\right)^{-1} \cdot V\left(\rho_{1}\left(\rho_{2}\right)^{k_{1}+k_{2}} \rho_{3}\right)
$$

Similarly from Equations 6.6 and 6.7 we get:

$$
V\left(\rho_{d, 3}^{\prime}\right)^{-1} \cdot V\left(\rho_{d, 3}\right)=V\left(\rho_{1}^{\prime}\left(\rho_{2}^{\prime}\right)^{k_{1}} \rho_{3}^{\prime}\right)^{-1} \cdot V\left(\rho_{1}\left(\rho_{2}\right)^{k_{1}} \rho_{3}\right)
$$

From Equations 6.9 and 6.10 we get:

$$
V\left(\rho_{1}^{\prime}\left(\rho_{2}^{\prime}\right)^{k_{1}+k_{2}} \rho_{3}^{\prime}\right)^{-1} \cdot V\left(\rho_{1}\left(\rho_{2}\right)^{k_{1}+k_{2}} \rho_{3}\right)=V\left(\rho_{1}^{\prime}\left(\rho_{2}^{\prime}\right)^{k_{1}} \rho_{3}^{\prime}\right)^{-1} \cdot V\left(\rho_{1}\left(\rho_{2}\right)^{k_{1}} \rho_{3}\right)
$$

By simplifying this expression we obtain:

$$
V\left(\left(\rho_{2}^{\prime}\right)^{k_{2}}\right)^{-1} \cdot V\left(\rho_{1}^{\prime}\right)^{-1} \cdot V\left(\rho_{1}\right) \cdot V\left(\left(\rho_{2}\right)^{k_{2}}\right)=V\left(\rho_{1}^{\prime}\right)^{-1} \cdot V\left(\rho_{1}\right)
$$




\begin{tabular}{|c|c|c|c|c|c|c|c|c|c|}
\hline \multirow[t]{2}{*}{ Decision Problems } & \multicolumn{3}{|c|}{ Sum/Avg-Automata } & \multicolumn{3}{|c|}{ Dsum-Automata } & \multicolumn{3}{|c|}{ Ratio-Automata } \\
\hline & D & $\mathrm{F}$ & ND & D & $\mathrm{F}$ & ND & $\mathrm{D}$ & $\mathrm{F}$ & ND \\
\hline functionality & - & - & $P$ & - & - & $\mathrm{P}$ & - & - & CoNP \\
\hline$>\nu$-emptiness & $P$ & $P$ & $\mathrm{P}[11$ & $P$ & $P$ & $P$ & $P$ & $\mathrm{P}$ & $P$ \\
\hline$\geq \nu$-emptiness & $P$ & $P$ & $\mathrm{P}[11]$ & $?$ & PSpace [9] & $?$ & $P$ & $\mathrm{P}$ & $P$ \\
\hline$>\nu$-universality & $P$ & $P$ & $\mathrm{U}[25,1]$ & $?$ & PSpace [9] & $?$ & $\mathrm{P}$ & $\mathrm{P}$ & $P$ \\
\hline$\geq \nu$-universality & $P$ & $P$ & $\mathrm{U}[25,1$ & $P$ & $\mathrm{P}$ & $P$ & $P$ & $\mathrm{P}$ & $P$ \\
\hline $\begin{array}{l}\text { inclusion } A \leq B \\
(A \text { arbitrary) }\end{array}$ & $P$ & PSpace-c & $\mathrm{U} 25$ & $P$ & PSpace-c & ? & D & D & $U$ \\
\hline equivalence & $P$ & PSpace-c & $\mathrm{U} 25$ & $P$ & PSpace-c & $?$ & D & $\mathrm{D}$ & U \\
\hline determinizability & - & CoNP & $?$ & - & CoNP & $?$ & - & $?$ & $?$ \\
\hline$>\nu$-realizability & $\begin{array}{l}\mathrm{NP} \cap \\
\mathrm{CoNP}\end{array}$ & U & $U$ & $\begin{array}{l}\mathrm{NP} \cap \\
\mathrm{CoNP}\end{array}$ & $?$ & $?$ & $\begin{array}{l}\mathrm{NP} \cap \\
\mathrm{CoNP}\end{array}$ & U & $U$ \\
\hline
\end{tabular}

Table 1: Complexity results for classes of weighted automata. In this table, D stands for deterministic, $\mathrm{F}$ for functional and ND for non-deterministic. The symbol $\mathrm{P}$ stands PTime, $U$ for undecidable, $\mathrm{D}$ for decidable and ? for open.

And therefore by multiplying by $V\left(\left(\rho_{2}^{\prime}\right)^{k_{1}}\right)^{-1}$ to the left and by $V\left(\left(\rho_{2}\right)^{k_{1}}\right)$ to the right, we obtain:

$$
\begin{aligned}
& V\left(\left(\rho_{2}^{\prime}\right)^{k_{1}+k_{2}}\right)^{-1} \cdot V\left(\rho_{1}^{\prime}\right)^{-1} \cdot V\left(\rho_{1}\right) \cdot V\left(\left(\rho_{2}\right)^{k_{1}+k_{2}}\right)= \\
& \quad=V\left(\left(\rho_{2}^{\prime}\right)^{k_{1}}\right)^{-1} \cdot V\left(\rho_{1}^{\prime}\right)^{-1} \cdot V\left(\rho_{1}\right) \cdot V\left(\left(\rho_{2}\right)^{k_{1}}\right)
\end{aligned}
$$

which can be rewritten as:

$$
V\left(\rho_{1}^{\prime}\left(\rho_{2}^{\prime}\right)^{k_{1}+k_{2}}\right)^{-1} \cdot V\left(\rho_{1}\left(\rho_{2}\right)^{k_{1}+k_{2}}\right)=V\left(\rho_{1}^{\prime}\left(\rho_{2}^{\prime}\right)^{k_{1}}\right)^{-1} \cdot V\left(\rho_{1}\left(\rho_{2}\right)^{k_{1}}\right)
$$

which is equivalent to $\Delta\left(k_{1}\right)=\Delta\left(k_{1}+k_{2}\right)$. This contradicts our hypothesis.

Therefore, we obtain:

Theorem 6.11. A functional infinitary group automaton is (effectively) determinizable iff it satisfies the twinning property. Determinizability is decidable in CoNP for functional infinitary group automata, and functional $V$-automata, for $V \in\{$ Sum, Avg, Dsum $\}$.

Proof. It follows directly from Lemmas 6.4 6.10 and Lemma 2.4.

\section{Conclusion}

In this paper, we have introduced and studied classes of functional weighted automata, for four measures: sum, average, discounted sum and ratio. Our results are summarized in Table 1. Despite our efforts, some problems remain open.

The determinizability of (non-deterministic) Sum-automata is a long standing open problem [22, 1]. Another notorious open problem, the so-called target discounted-sum problem [9], underlies a number of open questions related to Dsum automata and games: namely, e.g. imperfect information Dsum-games [15, multi-objective Dsum-games [12, universality of Dsum-automata [10], as well as the realizability problem for Dsum-automata that we mention here. Another open problem, that we did not mention so far, is to decide whether a (non-deterministic) Sum-automaton is equivalent to some functional Sum-automaton. 
Solving this problem would solve the determinizability problem for non-deterministic Sumautomata, as the determinizability problem for functional Sum-automata is decidable. However, this problem does not seem to be simpler than the determinizability problem. Finally, we leave the determinizability problem for functional Ratio-automata as open: the techniques we developed in this paper toward determinization are based on the well-known notion of delay between runs. It is not clear what would be a suitable notion of delays for Ratio-automata.

\section{ACKNOWLEDGEMENT}

We are very grateful to the anonymous referees for their valuable comments on preliminary versions of this paper.

\section{REFERENCES}

[1] S. Almagor, U. Boker, and O. Kupferman. What's decidable about weighted automata? In ATVA'11, pp 482-491. Springer, 2011.

[2] B. Aminof, O. Kupferman, and R. Lampert. Rigorous approximated determinization of weighted automata. In LICS, pp 345-354, 2011.

[3] D. Andersson. An improved algorithm for discounted payoff games. In ESSLLI Student Session, pp 91-98, 2006.

[4] M.-P. Beal, O. Carton, C. Prieur, and J. Sakarovitch. Squaring transducers: An efficient procedure for deciding functionality and sequentiality. TCS, 292, 2003.

[5] J. Berstel and C. Reutenauer. Rational Series and Their Languages. Number 12 in EATCS Monographs on TCS. Springer, 1988.

[6] M. Blattner and T. Head. Single-valued $a$-transducers. JCSS, 15(3), pp 310-327, 1977.

[7] R. Bloem, K. Greimel, T. A. Henzinger, and B. Jobstmann. Synthesizing robust systems. In FMCAD, pp 85-92. IEEE, 2009.

[8] U. Boker, K. Chatterjee, T. A. Henzinger, and O. Kupferman. Temporal specifications with accumulative values. In $L I C S$, pp 43-52, 2011.

[9] U. Boker, T. Henzinger and J. Otop. The Target Discount-Sum Problem. In LICS. To appear, 2015.

[10] U. Boker and T. A. Henzinger. Determinizing discounted-sum automata. In CSL, pp 82-96, 2011.

[11] K. Chatterjee, L. Doyen, and T. A. Henzinger. Quantitative languages. ACM Trans. Comput. Log, 11(4), 2010.

[12] K. Chatterjee, V. Forejt, and D. Wojtczak. Multi-objective Discounted Reward Verification in Graphs and MDPs. Logic for Programming, Artificial Intelligence, and Reasoning, LNCS 8312, 2013.

[13] C. Choffrut. Une caractérisation des fonctions séquentielles et des fonctions sous-séquentielles en tant que relations rationnelles. TCS, 5(3), pp 325-337, 1977.

[14] L. de Alfaro, M. Faella, T. A. Henzinger, R. Majumdar, and M. Stoelinga. Model checking discounted temporal properties. TCS, 345(1), pp 139-170, 2005.

[15] A. Degorre, L. Doyen, R. Gentilini, J-F. Raskin and S. Torunczyk Energy and Mean-Payoff Games with Imperfect Information. In CSL-2010, LNCS vol. 6247, pp 260-274, 2010.

[16] M. Droste, W. Kuich, and H. Vogler. Handbook of Weighted Automata. Springer, 2009.

[17] M. Droste and G. Rahonis. Weighted automata and weighted logics with discounting. In CIAA, pp 73-84, 2007.

[18] A. Ehrenfeucht and J. Mycielski. Positional strategies for mean payoff games. In International Journal of Game Theory, 8(2):109113, 1979.

[19] E. Filiot, R. Gentilini and J-F. Raskin Quantitative languages defined by functional automata. In CONCUR-2012, LNCS vol. 7454, pp 132-146, 2012.

[20] F. Grunewald and D. Segal. On the integer solutions of quadratic equations. Journal für die reine und angewandte Mathematik, 569, pp 13-45, 2004.

[21] E. Gurari and O. Ibarra. A note on finitely-valued and finitely ambiguous transducers. Mathematical Systems Theory, 16(1):61-66, 1983. 
[22] D. Kirsten. A burnside approach to the termination of mohri's algorithm for polynomially ambiguous min-plus-automata. ITA, 42(3), pp 553-581, 2008.

[23] D. Kirsten and I. Mäurer. On the determinization of weighted automata. Journal of Automata, Languages and Combinatorics, 10(2/3), pp 287-312, 2005.

[24] I. Klimann, S. Lombardy, J. Mairesse, and C. Prieur. Deciding unambiguity and sequentiality from a finitely ambiguous max-plus automaton. TCS, 327(3), pp 349-373, 2004.

[25] D. Krob. The equality problem for rational series with multiplicities in the tropical semiring is undecidable. Journal of Algebra and Computation, 4(3), pp 405-425, 1994.

[26] D. Krob and P. Litp. Some consequences of a fatou property of the tropical semiring. J. Pure Appl. Algebra, 93, pp 231-249, 1994.

[27] S. Lombardy and J. Mairesse. Series which are both max-plus and min-plus rational are unambiguous. ITA, 40(1), pp 1-14, 2006.

[28] M. L. Minsky. Computation: Finite and Infinite Machines. Prentice-Hall, 1967.

[29] M. Mohri. Finite-State Transducers in Language and Speech Processing. Computational Linguistics, pp 269-311, 1997.

[30] M. Mohri. Weighted automata algorithms. Handbook of Weighted Automata, pp 213-254, 2009.

[31] R. J. Parikh On context-free languages. Journal of the ACM, 13 (4) (1966) 570581.

[32] A. Pnueli and R. Rosner. On the synthesis of a reactive module. In ACM Symposium on Principles of Programming Languages (POPL). ACM, 1989.

[33] E. M. Schmidt and T. G. Szymanski. Succinctness of descriptions of unambiguous context-free languages. In SIAM Journal on Computing, 6(3), pp 547-553, 1977.

[34] M. P. Schützenberger. Sur les relations rationnelles. In Automata Theory and Formal Languages, vol 33 of $L N C S$, pp 209-213, 1975.

[35] W. Thomas. Church's problem and a tour through automata theory. In Pillars of Computer Science, pp 635-655, 2008.

[36] A. Weber. On the valuedness of finite transducers. Acta Informatica, 27(8):749-780, 1989.

[37] A. Weber and R. Klemm. Economy of description for single-valued transducers. Inf. Comput., 118(2), pp 327-340, 1995.

[38] K. Wong, A. Krieg, and W. Thomas. On intersection problems for polynomially generated sets. In ICALP, vol 4052 of $L N C S$, pp 516-527. Springer, 2006.

[39] U. Zwick and M. Paterson. The complexity of mean payoff games on graphs. In Theoretical Computer Science, 158(1-2):343359, 1996. 